NBER WORKING PAPER SERIES

\title{
LINKING CHANGES IN INEQUALITY IN LIFE EXPECTANCY AND MORTALITY: EVIDENCE FROM DENMARK AND THE UNITED STATES
}

\author{
Gordon Dahl \\ Claus Thustrup Kreiner \\ Torben Heien Nielsen \\ Benjamin Ly Serena \\ Working Paper 27509 \\ http://www.nber.org/papers/w27509
NATIONAL BUREAU OF ECONOMIC RESEARCH
1050 Massachusetts Avenue
Cambridge, MA 02138
July 2020

We thank participants at the NBER workshop on Income and Life Expectancy (2019) in Boston, the workshop on Health Inequalities (2019) at the Copenhagen Business School and the workshop on Behavioral Responses to Health Innovations and the Consequences for Socioeconomic Outcomes (2019) at the University of Copenhagen for helpful discussions and comments. We are also grateful for discussions with Bo Honore and Chris Ruhm. Kristian Urup Olesen Larsen provided excellent research assistance. The Center for Economic Behavior and Inequality (CEBI) at the University of Copenhagen is supported by Danish National Research Foundation Grant DNRF134. This research was also supported by Novo Nordisk Foundation Grant NNF17OC0026542. The views expressed herein are those of the authors and do not necessarily reflect the views of the National Bureau of Economic Research.

NBER working papers are circulated for discussion and comment purposes. They have not been peer-reviewed or been subject to the review by the NBER Board of Directors that accompanies official NBER publications.

(C) 2020 by Gordon Dahl, Claus Thustrup Kreiner, Torben Heien Nielsen, and Benjamin Ly Serena. All rights reserved. Short sections of text, not to exceed two paragraphs, may be quoted without explicit permission provided that full credit, including $\odot$ notice, is given to the source. 
Linking Changes in Inequality in Life Expectancy and Mortality: Evidence from Denmark and the United States

Gordon Dahl, Claus Thustrup Kreiner, Torben Heien Nielsen, and Benjamin Ly Serena

NBER Working Paper No. 27509

July 2020

JEL No. H51,I14,J11

\section{ABSTRACT}

We decompose changing gaps in life expectancy between rich and poor into differential changes in age-specific mortality rates and differences in "survivability". Declining age-specific mortality rates increases life expectancy, but the gain is small if the likelihood of living to this age is small (ex ante survivability) or if the expected remaining lifetime is short (ex post survivability). Lower survivability of the poor explains half of the recent rise in life expectancy inequality in the US and the entire rise in Denmark. Cardiovascular mortality declines favored the poor, but differences in lifestyle-related survivability led inequality to rise.

Gordon Dahl

Department of Economics

University of California, San Diego

9500 Gilman Drive \#0508

La Jolla, CA 92093-0508

and NBER

gdahl@ucsd.edu

Claus Thustrup Kreiner

Department of Economics

University of Copenhagen and CEBI

Øster Farimagsgade 5, Building 35

DK-1353 Copenhagen K

Denmark

claus.thustrup.kreiner@econ.ku.dk
Torben Heien Nielsen

University of Copenhagen

Department of Economics

Øster Farimagsgade 5, Building 35

DK-1353 Copenhagen K

Denmark

torben.heien.nielsen@econ.ku.dk

Benjamin Ly Serena

Department of Economics

University of Copenhagen and CEBI

Øster Farimagsgade 5, Building 35

DK-1353 Copenhagen K

Denmark

Benjamin.Ly.Serena@econ.ku.dk 
Life expectancy is strongly associated with socioeconomic status. This is a fundamental aspect of inequality in society with important implications for the progressivity of public health and social security policies (Marmot et al., 2010; Poterba, 2014; Auerbach et al., 2017). In many OECD countries, inequality in life expectancy has been rising (Waldron, 2007; Case and Deaton, 2015; Chetty et al., 2016; Currie and Schwandt, 2016; Auerbach et al., 2017; Bor et al., 2017; Hederos et al., 2017; Kreiner et al., 2018; Kinge et al., 2019). Figure 1 displays estimates of life expectancy at age 40 across income tertiles for males and females in the United States and Denmark for 2001 and 2014. ${ }^{1}$ The estimates are based on a standard computation of period life expectancy by social class using population-wide register data on mortality and income (see Chetty et al. 2016). The gap in life expectancy between rich (top tertile) and poor (bottom tertile) males is around 8 years in both the US and Denmark in 2001. Over the short period from 2001 to 2014, this inequality in life expectancy increased by 1.7 years in the US and 0.9 years in Denmark. The gap in life expectancy between rich and poor females stayed constant in Denmark over this period, but also increased by about 1.8 years in the US. $^{2}$

The driving forces behind the recent trends in life expectancy inequality remain unclear. Several studies focus on comparing changes in age-specific mortality rates by socioeconomic status (LlerasMuney, 2005; Snyder and Evans, 2006; Cutler et al., 2011; van den Berg et al., 2017; Mackenbach et al., 2018; Baker et al., 2019; Montez et al., 2019; Attanasio and Nielsen, 2020), but how do these changes translate into trends in life expectancy inequality? We link the dynamics of life expectancy inequality to the underlying dynamics of mortality inequality by decomposing the changes in life

\footnotetext{
${ }^{1}$ We focus on life expectancy at age 40 following recent work estimating its income gradient (e.g., Chetty et al., 2016; Kreiner et al., 2018; Kinge et al., 2019). Other research looks at life expectancy at birth. This approach includes changes in mortality inequality at younger ages, which in isolation have reduced life expectancy in recent decades in some countries (Currie and Schwandt, 2016; Baker et al., 2019). At birth the income of an individual is not yet realized and, therefore, these studies typically analyze differences in mortality and income across geographical areas.

${ }^{2}$ The higher life expectancy for the US compared to Denmark reflects that the US data excludes individuals with zero earnings, while the Danish data covers the full population. Conclusions are unchanged if we impose a similar restriction on the Danish data to make estimates comparable (see the Appendix).
} 
expectancy inequality into differential mortality trends between rich and poor and a common trend. This common trend affects life expectancy inequality through differences in "survivability". 3

Survivability is a summary measure of initial age-specific mortality rates and has a simple interpretation. Survivability at a given age measures the likelihood of surviving until this age multiplied by the expected remaining life years after surviving this age. Intuitively, a person only benefits from a reduction in an age-specific mortality rate if they have survived until this age (ex ante effect) and, if so, the benefit is the expected extra life years thereafter (ex post effect).

An increasing gap in life expectancy by income class may arise because mortality rates decline more for the rich than the poor, for example if new health technologies differentially benefit the rich (Cutler et al., 2006; Jayachandran et al., 2010; Cutler et al., 2011; Moscelli et al., 2018). But it may also arise because a given drop in mortality rates leads to larger increases in life expectancy of the rich due to their higher survivability. Survivability is highest for the rich due to lower initial mortality rates. As we show, this implies somewhat paradoxically that the gap in life expectancy between the rich and poor can increase, even if the gap in mortality rates is constant or declining. Thus, both differential mortality rate changes across groups and existing mortality inequality across groups (which translates into survivability differences) are key determinants of changes in life expectancy inequality over time.

Empirically, we find that it is important to account for survivability when evaluating changes in life expectancy inequality in both the US and Denmark. In the US, half of the rise in inequality for forty-year old males shown in Figure 1 is due to larger reductions in mortality rates for the rich than the poor, while the other half is due to differences in their survivability. For Danish males in Figure 1, life expectancy inequality increased, even though mortality rates have fallen more for the poor. The explanation for this apparent puzzle is that survivability strongly favored the rich, more than

\footnotetext{
${ }^{3}$ Many decomposition methods and applications exist in demography, economics, and other social sciences as surveyed in Canudas Romo (2003), but as far as we know, nobody has decomposed life expectancy between groups and over time into these two components before.
} 
offsetting the effect of differential mortality rate changes. For females in both countries, survivability plays a similarly important role. In the US, one-third of the rise in inequality for females is due to survivability. For females in Denmark, there is virtually no change in life expectancy inequality. Even though drops in mortality in isolation added an extra life year to poor relative to rich Danish females, this is fully offset by the higher survivability of the rich.

Motivated by recent influential work, we next explore how changes in cause-specific mortality have interacted with cause-specific survivability. Case and Deaton (2015) document unequal increases in deaths caused by drug and alcohol poisonings, suicide, chronic liver diseases and cirrhosis, which are all indicative of adverse health behaviors. Chetty et al. (2016) and Kinge et al. (2019) likewise associate life expectancy gaps with differences in health behaviors such as smoking and drinking. More broadly, cardiovascular disease and cancer remain the main causes of deaths in developed economies. However, while cancer deaths only saw modest improvement between 2001 to 2014, deaths due to cardiovascular disease dropped by 50\%. Cutler and Kadiyala (2003) attribute one-third of the improvement in cardiovascular mortality since the 1950s to improved behavioral technologies, such as reduced smoking and better nutrition, and the remaining two-thirds to invasive treatment and pharmaceutical innovations.

We, therefore, extend our decomposition of life expectancy inequality into four broad death categories: cardiovascular, behavioral (following Case and Deaton, 2015), cancers, and other causes. ${ }^{4}$ In Denmark, where we are able to link cause-specific deaths to income, we observe a large drop in cardiovascular mortality rates, and more so for the poor than the rich. Despite the larger reduction in mortality of the poor, the gap in life expectancy increased because of lower survivabililty of the poor, who have higher risks of dying from lifestyle-related diseases (such as liver disease and lung cancer). This shows that widening inequality did not happen because behavioral mortality

\footnotetext{
${ }^{4}$ We group causes of death in these four categories to enable easier comparisons to the existing literature, while also recognizing that some of the cardiovascular and cancer deaths are behaviorally related.
} 
differentially worsened for the poor, but because reductions in cardiovascular deaths made existing differences in behavioral mortality more consequential. Defining socioeconomic class by education level instead of income allows us to perform an analogous exercise for the US, where a similar conclusion emerges.

Our decomposition results demonstrate the value of linking the dynamics of life expectancy inequality to the underlying dynamics in age-specific mortality rates. Trends in age-specific mortality rates of the rich and poor are informative about changes in underlying health status, and trends in life expectancy are a relevant measure of the associated welfare effects. Looking at each of these in isolation misses an important link - survivability - and, as demonstrated by our empirical results, can lead to misleading conclusions. Moreover, our cause-specific findings make clear that the driving forces for changes in mortality inequality can be very different from those for changes in life expectancy inequality.

The remainder of the paper proceeds as follows. Sections 1 to 3 provide an illustrative example, followed by our decomposition formulas, and a description of our data. Section 4 documents the empirical importance of differences in survivability. Section 5 evaluates contributions from causespecific mortality. Section 6 discusses a variety of possible extensions and robustness checks. Section 7 concludes.

\section{An Illustrative Empirical Example}

To illustrate the importance of survivability for changes in life expectancy inequality, consider the impact of the observed change in one-year mortality rates for 60-year old males between 2001 and 2014 on life expectancy at age 40. Column 1 of Table 1 shows these changes for the rich and poor in both the US and Denmark. While the decrease in mortality at age 60 is the same across the income groups within each country, the impact on life expectancy is not. The reason is survivability. In the US, $95 \%$ of 40 -year-old males are predicted to survive to age 60 if they are rich, compared to $84 \%$ 
of the poor (column 2). Hence, poor individuals are less likely than rich individuals to survive long enough to benefit from the reduction in mortality which occurs at age 60 (ex ante survivability). For those who do survive past age 60, remaining life expectancy is 23.5 years for the rich versus 19.0 for the poor (column 3). In other words, the poor are more likely to die sooner if they survive to age 61 compared to the rich (ex post survivability).

By multiplying the two survivability components, we obtain the total survivability effect in column 4 . The rich gain 22.3 years if the mortality rate goes from 1 to 0 at age 60 , while the poor only gain 16.0 years. Finally, by multiplying the observed changes in mortality (column 1) with the survivability (column 4), we obtain the change in life expectancy in column 5 . This calculation reveals that even with the same change in mortality (-.002) for the rich and the poor, the rich gain 1.4 times more years of life expectancy (.045 versus .032 years). A similar pattern holds for Denmark. It further follows from the example (by continuity) that it is possible for mortality to decline more for the poor than the rich, thereby narrowing the gap in mortality, while at the same time have the gap in life expectancy expand. Indeed, this is what happens in practice for Denmark, as we show below.

The example in Table 1 illustrates how changes in mortality rates at a given age map into changes in life expectancy and the role played by survivability. The next section shows this more generally with mathematical formulas that are later used to assess the empirical importance of survivability.

\section{Decomposition Formulas}

A standard formula for life expectancy measured at age $\underline{a}$ is

$$
\begin{aligned}
L E_{\underline{a}} & =\underline{a}+\left(1-M_{\underline{a}}\right)+\left(1-M_{\underline{a}}\right)\left(1-M_{\underline{a}+1}\right)+\ldots \\
& =\underline{a}+\sum_{a=\underline{a}}^{\bar{a}} \prod_{i=\underline{a}}^{a}\left(1-M_{i}\right)
\end{aligned}
$$


where $M_{a}$ is the mortality rate at age $a$ and $\bar{a}$ is the maximum age. This equation and the following decomposition formulas apply both to period life expectancy and cohort life expectancy, the difference being how age-specific mortality rates are estimated. Cohort life expectancy uses mortality of the same cohorts over time, while period life expectancy uses mortality of different cohorts in a given period. In our empirical application, we focus on period life expectancy in line with recent studies of inequality over time (Chetty et al., 2016; Currie and Schwandt, 2016; Hederos et al., 2017; Kreiner et al., 2018; Kinge et al., 2019). ${ }^{5}$

Differentiating equation (1) with respect to mortality rates and summing over all ages yields the following first-order approximation for the change in life expectancy (see Appendix Section 3):

$$
\Delta L E_{\underline{a}} \approx-\sum_{a=\underline{a}}^{\bar{a}} \Delta M_{a} \cdot X_{a} \text { where } X_{a} \equiv S_{a} \cdot R_{a}
$$

where $S_{a} \equiv \prod_{i=\underline{a}}^{a-1}\left(1-M_{i}\right)$ is the probability of survival from age $\underline{a}$ to age $a$ and $R_{a} \equiv 1+$ $\sum_{j=a+1}^{\bar{a}} \prod_{i=a+1}^{j}\left(1-M_{i}\right)$ is remaining life expectancy after surviving age $a$, in which case the individual lives one extra year for sure and from age $a+1$ can expect to live the additional years given by the last term in the definition.

Equation (2) expresses the change in life expectancy as the product of changes in mortality rates $\left(\Delta M_{a}\right)$ and survivability $\left(X_{a}\right)$, where survivability is a summary measure of initial mortality rates that includes the ex ante $\left(S_{a}\right)$ and ex post $\left(R_{a}\right)$ terms. This aligns with the intuition from Table 1, the only difference being that the formula sums the changes over all possible ages. Equation (2) is a mathematical identity for infinitesimal mortality changes and a first-order approximation for actual changes. ${ }^{6}$

\footnotetext{
${ }^{5}$ Since period life expectancy is a summary measure of cross-sectional mortality rates at a given point in time, it is often used to study trends in inequality. In a steady state with constant age-specific mortality rates, period life expectancy would equal the observed average life length. Therefore, comparing period life expectancy at two points in time, as done in the literature, is effectively comparing expected longevity between two (artificial) steady states and does not, for example, capture the full benefits on actual longevity from health improvements that take place in this time span. For a further discussion of period life expectancy and cohort life expectancy see Guillot (2011).

${ }^{6}$ This is similar to the Arriaga age decomposition often used in demography (Arriaga, 1984). In addition to the first-order approximation (2), the Arriaga approximation includes an extra term, an interaction effect, equal to
} 
By applying equation (2) for rich and poor and using a little algebra (see Appendix Section 3), we obtain the following decomposition formula for the change in life expectancy inequality between the rich (superscript $r$ ) and the poor (superscript $p$ ):

$$
\Delta L E_{\underline{a}}^{r}-\Delta L E_{\underline{a}}^{p} \approx \sum_{a=\underline{a}}^{\bar{a}} \overline{X_{a}} \cdot\left(\Delta M_{a}^{p}-\Delta M_{a}^{r}\right)+\sum_{a=\underline{a}}^{\bar{a}} \overline{\Delta M_{a}} \cdot\left(X_{a}^{p}-X_{a}^{r}\right)
$$

where $\overline{X_{a}}$ is the average survivability of the rich and the poor, while $\overline{\Delta M_{a}}$ is the average change in their mortality rates. The formula decomposes the change in life expectancy inequality into two terms. The first term isolates the contribution from differential changes in the mortality rates of rich and poor, holding survivability constant at the average across income groups $\left(X_{a}^{r}=X_{a}^{p}=\overline{X_{a}}\right)$. In other words, this term measures the effect of changing mortality inequality on life expectancy inequality in a counterfactual situation where initial mortality rates/survivability are identical for rich and poor. The second term isolates the contribution from differences in survivability between the rich and poor, holding mortality rate changes constant across the two groups. Thus, it measures the change in life expectancy inequality in a counterfactual situation where changes in mortality rates are the same for the rich and poor $\left(\Delta M_{a}^{r}=\Delta M_{a}^{p}=\overline{\Delta M_{a}}\right)$, in which case the change in life expectancy inequality is driven entirely by the differences in survivability.

In our illustrative empirical example from the US and Denmark in Section 1, the changes in mortality rates were the same for the rich and poor, implying the change in life expectancy inequality was driven by differences in survivability (the first term in the formula is zero). The opposite special case would arise if mortality rates of the two groups moved in opposite directions so that the average change in the mortality rates was zero $\left(\overline{\Delta M_{a}}=0\right)$. In this case, the change in life expectancy

$\Delta R_{a} \cdot \Delta M_{a} \cdot S_{a}$. The interaction effect cannot be attributed to any particular age, but reduces the approximation error of the age decomposition. Appendix Section 4 demonstrates that the first-order approximation (2) is fairly accurate in our empirical application, and that the main results based on the decomposition formula (3) are unchanged if we use an Arriaga approximation. 
inequality would be driven solely by the change in mortality inequality (the second term in the formula is zero).

Our decomposition is related to the seminal work of Kitagawa (1955). The general Kitagawa decomposition method has been applied and extended in various forms in demography, economics, and other social sciences. ${ }^{7}$ Normally, the purpose is to decompose the difference in crude rates (e.g., crude death rates) between two populations into differences in the composition of characteristics in the population and differences in characteristic-specific rates. Our decomposition links changes in life expectancy inequality to differential mortality trends between rich and poor, and the overall mortality trend, which affects life expectancy inequality through differences in survivability. To our knowledge, nobody has decomposed life expectancy inequality between groups and over time in this way before.

The Oaxaca-Blinder decomposition widely used in economics is a related idea, and decomposes the difference in outcomes between two groups into differences in mean characteristics across the groups and the differential effect of characteristics across groups (Oaxaca, 1973; Blinder, 1973). Technically, our decomposition differs from the standard Oaxaca-Blinder decomposition, in part because it sums over a variety of ages, and in part because it uses the combined means for both groups $\left(\overline{X_{a}}\right.$ and $\overline{\Delta M_{a}}$, rather than separate means by group). We further take advantage of the fact that with two equally-sized groups, we can use the combined means for both groups without needing to account for any covariance terms in the decomposition formula.

\footnotetext{
${ }^{7}$ See Canudas Romo (2003)) for a detailed review of various decomposition methods and applications in demography. See Fortin et al. (2011) for a discussion of decomposition methods in economics.
} 
Using the definition $X_{a} \equiv S_{a} \cdot R_{a}$, we can further decompose the last term in equation (3) into the contributions from ex ante and ex post survivability, and arrive at the following decomposition:

$$
\begin{aligned}
\Delta L E_{\underline{a}}^{r}-\Delta L E_{\underline{a}}^{p} \approx & \underbrace{\sum_{a=\underline{a}}^{\bar{a}} \overline{X_{a}} \cdot\left(\Delta M_{a}^{p}-\Delta M_{a}^{r}\right)}_{\Delta \text { Mortality }} \\
& +\underbrace{\sum_{a=\underline{a}}^{\bar{a}} \overline{\Delta M_{a}} \cdot \overline{R_{a}} \cdot\left(S_{a}^{p}-S_{a}^{r}\right)}_{\text {Ex ante survivability }}+\underbrace{\sum_{a=\underline{a}}^{\bar{a}} \overline{\Delta M_{a}} \cdot \overline{S_{a}} \cdot\left(R_{a}^{p}-R_{a}^{r}\right)}_{\text {Ex post survivability }}
\end{aligned}
$$

where $\overline{R_{a}}$ and $\overline{S_{a}}$ are the average ex ante and ex post survivability of the rich and the poor.

\section{Data}

Our empirical analysis is based on two separate data sources with information about mortality for the US and Denmark from 2001 to 2014. US data on mortality by income class and age is from the study by Chetty et al. (2016) and is computed by the authors based on the universe of tax returns of the US population. This data set is publicly available at www.opportunityinsights . org/data. Using population-wide administrative registers provided by Statistics Denmark, we computed mortality by income class, age, and cause of death for Denmark. We have made our aggregate data available online. All results in the main text can be replicated from these two publicly available data sets using Stata code provided online. ${ }^{8}$

The US data differs in important ways from the Danish data. While the Danish data covers the entire population, the US data does not include individuals with zero earnings, many of whom are disability insurance recipients. This sample restriction drops $9 \%$ of the US population and $32 \%$ of deaths. Therefore, mortality rates for the US are underestimated and life expectancies are overestimated compared to population averages. In Appendix A.2, we show that the conclusions are

\footnotetext{
${ }^{8}$ The individual-level tax return data used by Chetty et al. (2016), underlying the US data set, is confidential. Similarly, the underlying individual-level information from the Danish administrative registers is confidential.
} 
unchanged if we exclude individuals with zero or negative income and disability insurance recipients from the Danish data, to make results for Denmark and the US more comparable.

A second advantage of the Danish data is that it is not necessary to impute mortality rates by socioeconomic status past retirement (Kreiner et al., 2018). The US income measure includes gross income minus Social Security and disability benefits. The Danish income measure includes gross income minus universal benefits (public pension, disability pension), but including pension payouts from private and employer-based pension accounts. These payouts are tightly linked to previous labor market earnings and, therefore, the income concept remains a good measure of socioeconomic status after retirement. In the US data, income in retirement is less correlated with previous earnings and Chetty et al. (2016) therefore impute mortality rates by socioeconomic status after retirement using Gompertz approximations.

A third advantage of the Danish data is that cause of death can be linked to income data. This facilitates our cause-specific decomposition of changes in life expectancy inequality. In the US, this type of analysis is not possible. However, using data from Case and Deaton (2017) we can link cause of death to education level in the US, which the Danish register data also allows for. Using education as a proxy of socioeconomic status instead of income is not ideal, because education levels are coarse and because educational distributions change over time. However, it does allow us to present cause-specific decompositions of the change in life expectancy inequality for both the US and Denmark.

Additional information about the data sets, construction of the variables, empirical methodology, and supplementary analyses are provided in the Appendix.

\section{Empirical Importance of Differences in Survivability}

The simple empirical example for the US and Denmark provided in Table 1 illustrates the role of survivability for rising life expectancy inequality. For both countries, the change in mortality at 
age 60 is identical for the rich and the poor. However, this is not true at all ages. The left panel of Figure 2 plots the change in mortality from 2001-2014 over 5-year age bins for the rich and the poor. An interesting contrast emerges between the two countries. In the US, starting around age 60, mortality rates fall more dramatically for the rich than the poor for both males and females. In Denmark, the drop in age-specific mortality rates has, for most ages, actually favored the poor more than the rich.

The right panel plots survivability, which is the product of survival until a given age (ex ante survivability) multiplied by remaining life expectancy beyond that age (ex post survivability). Survivability always favors the rich, both in the US and Denmark and for both males and females. While not shown in the figure, both ex ante and ex post survivability favor the rich at every age. In other words, the rich are more likely to be alive to benefit from a drop in mortality at any age, and have more remaining years to benefit at any age. The gap in survivability is largest for males and narrows with age, but it never completely disappears. This means that a common drop in mortality rates at any age will mechanically favor the rich, widening inequality in life expectancy.

To assess the contributions of each component more systematically, we use the decomposition formulas (3) and (4). Focusing first on the US, we decompose the change in inequality in life expectancy between the rich and poor from 2001 to 2014. As can be seen in Figure 3, for males, the gap in life expectancy between the rich and poor increased in total by approximately 1.4 years (blue bar). Roughly half of this increase is attributable to larger drops in mortality for the rich than the poor (green bar). The other half is due to survivability which favors the rich, with ex ante and ex post survivability playing equal roles (red bars). ${ }^{9}$

\footnotetext{
${ }^{9}$ One way to benchmark the contribution of ex ante versus ex post survivability is to calculate the effect survivability would have if the overall decline in mortality across all ages instead had been concentrated at a single, specific age.. For US males, if the entire mortality decline was concentrated at age 40, there would be a 1.5 year increase in life expectancy inequality between the rich and poor. All of the effect would operate through ex post survivability since there are no differences in ex ante survivability at age 40. If the entire mortality decline was instead concentrated at age 85 , there would have been a 0.4 year increase in life expectancy inequality, with almost all of the gap being driven by ex ante survivability. These benchmarks compare to the actual survivability effect of a 0.7 year increase, and indicate that a general reduction in mortality has a larger effect if it occurs at younger ages.
} 
For females in the US, there is a 1.5-year increase in life expectancy inequality. Over two-thirds of this rise is attributable to drops in mortality favoring the rich. The remaining is mostly due to ex ante survivability, i.e., a larger fraction of poor females not living long enough to benefit from reductions in mortality.

A different pattern emerges for Denmark in Figure 3, where age-specific improvements in mortality have differentially favored the poor. For males, changes in mortality reduce inequality by a sizeable 0.8 years. In spite of this, overall life expectancy inequality increases by 0.7 years. This reversal in sign occurs because survivability strongly favors the rich. Ex ante survivability accounts for a 0.9-year increase in life expectancy inequality, and ex post survivability accounts for another 0.6-year increase. So while drops in age-specific mortality rates favored the poor, the poor did not live long enough to benefit and did not gain as many years of remaining life compared to the rich.

For females in Denmark, there is virtually no change in life expectancy inequality. This is despite the fact that drops in mortality contributed almost a full year more to life expectancy for the poor than the rich during this 14-year period. But offsetting the drop in mortality favoring the poor was an equally large contribution from survivabililty favoring the rich. Ex ante and ex post survivability each account for roughly a half-year increase in life expectancy inequality.

The differential changes in life expectancy by socioeconomic status for females versus males leads naturally to the question of how the female-male gap in life expectancy has evolved over this same time period. Females have longer life expectancies than males, but the gap has decreased in recent years (Goldin and Lleras-Muney, 2019). Life expectancy of females exceeded that of males by 4.1 years in 2001 in the US, but the difference fell by 0.4 years to 3.7 by 2014 . The female-male gap in life expectancy fell by a similar amount in Denmark, from 4.0 years to 3.6 years over this same time period. 
We use our decomposition formula (3), but applied to females and males instead of rich and poor, to assess the importance of survivability. Appendix Figure A.9 illustrates the decomposition. It shows that changes in mortality rates declined faster for males versus females between 2001 and 2014, with almost a full-year decline in the US and a 1.2-year decline in Denmark. But counteracting this drop was lower survivability for males. In other words, lower ex ante and ex post survivability (the dark and light red bars) meant that males did not live long enough to benefit from the differential change in mortality and had fewer remaining life years upon surviving a given age. Had males' survivability matched that of females', the gender gap would have narrowed three times more.

\section{Contributions from Cause-Specific Mortality}

The previous section highlights the importance of survivability for understanding changes in life expectancy inequality. We now explore how recent changes in cause-specific mortality have interacted with cause-specific survivability to affect life expectancy inequality. To do this, we extend our decomposition approach so that the components of equation (4) are cause specific, where the subscript $c$ denotes the cause (see Appendix Section 5): ${ }^{10}$

$$
\begin{aligned}
\Delta L E_{\underline{a}}^{r}-\Delta L E_{\underline{a}}^{p} \approx & \underbrace{\sum_{a=\underline{a}}^{\bar{a}} \bar{X}_{a} \cdot \sum_{c}\left(\Delta M_{a, c}^{p}-\Delta M_{a, c}^{r}\right)}_{\Delta \text { Mortality }} \\
& +\underbrace{\sum_{a=\underline{a}}^{\bar{a}} \overline{\Delta M_{a}} \cdot \overline{R_{a}} \cdot \sum_{c}\left(S_{a, c}^{p}-S_{a, c}^{r}\right)}_{\text {Ex ante survivability }}+\underbrace{\sum_{a=\underline{a}}^{\bar{a}} \overline{\Delta M_{a}} \cdot \overline{S_{a}} \cdot \sum_{c}\left(Q_{j, c}^{r}-Q_{j, c}^{p}\right)}_{\text {Ex post survivability }}
\end{aligned}
$$

where $\Delta M_{a, c}^{r}$ and $\Delta M_{a, c}^{p}$ are cause-specific mortality rates of the rich and poor, $S_{a, c}^{r}$ and $S_{a, c}^{p}$ are their probabilities of not dying of cause $c$ before age $a$, and $Q_{a, c}^{r}$ and $Q_{a, c}^{p}$ denote their expected lost life measured in years from age $a$ to $\bar{a}$ due to the risks of dying of cause $c$.

\footnotetext{
${ }^{10}$ This cause-specific decomposition of changes in life expectancy inequality is novel. Note that it deviates from an Arriaga decomposition, which decomposes life expectancy by cause-specific mortality differences (Kinge et al., 2019; Ho and Hendi, 2018) either between groups or over time, and which attributes age-specific contributions based on cause-specific mortality differences at that age. Our decomposition allows us to study differences in life expectancy between groups and over time, and captures that the impact of cause-specific changes in mortality rates at one age depend on initial differences in cause-specific mortality at other ages (cause-specific survivability).
} 
The first term in the formula reflects that the rich might experience larger declines in mortality rates from death cause $c$ than the poor, which increases the gap in life expectancy between the two groups for a given level of survivability. The second term reflects that a common decline in overall mortality rates of the rich and poor at a given age $a$ may lead to higher inequality in life expectancy because the poor are more likely to have died from death cause $c$ before reaching age $a$. The third term reflects that a common decline in overall mortality rates for the rich and poor at a given age $a$ may lead to higher inequality in life expectancy because the poor are more likely to die from death cause $c$ in the future. As a consequence, the expected lost lifetime after age $a$, due to death cause $c$, is larger for the poor, who therefore benefit less from the common reduction in the mortality rate at age $a$.

The key assumption needed for this type of decomposition is independence across cause-specific changes in mortality rates. This simplifying assumption, which is generally made in cause-specific decompositions of life expectancy and in related work on competing risks, implies that improvements in cardiovascular mortality rates do not, for example, affect changes in cancer mortality rates. One exception is Honoré and Lleras-Muney (2006), which provides bounds in a competing risk model without assuming independence. They estimate changes in cancer and cardiovascular mortality over time without assuming independence, and find that the improvements in cancer are larger compared to estimates which assume independence.

We decompose changes in life expectancy inequality into four broad categories: cardiovascular, behavioral, cancers, and other causes. The separation into these four categories is motivated by cardiovascular and cancers being leading causes of death, and because what we label as "behavioral" has been the focus of influential research. Figure 4 shows time trends in these four cause-specific mortality rates for Denmark and the US. The red curve reveals a dramatic reduction in cardiovascular mortality in both countries. This represents a global trend, which has been associated 
with medical advances in the treatment and prevention of cardiovascular disease (Deaton, 2013; Cutler and Kadiyala, 2003; Likosky et al., 2018). The blue line plots the trend for what we label "behavioral" causes and is constructed using similar causes of death as in Case and Deaton (2015). Behavioral causes include external causes (suicides, homicides, poisonings, accidents), liver and gallbladder diseases, respiratory cancers, bronchitis and asthma, substance abuse, and diabetes. ${ }^{11}$ The yellow line includes all cancers but respiratory cancers, and the green line groups all other causes. While mortality rates for both cancer and behavioral causes decline modestly over our sample period, these drops are overshadowed by the halving of cardiovascular mortality.

Figure 5 plots the cause-specific decomposition results for Denmark based on income groups. The gray bars in Figure 5 replicate the decomposition already presented for Danish males and females in Figure 3. The colored bars further decompose each of the gray bars into contributions due to the different causes of death. Similar data on cause-specific mortality by income is not readily available for the US, so later we alternatively use education as a proxy for social status for both countries and perform similar cause-specific decompositions.

We first look at the importance of cause-specific mortality rate changes for rich versus poor Danish males. The red bar shows that the big drop in cardiovascular mortality contributed to a large decline of 0.7 years in the rich-poor life expectancy gap. This is the main reason why changes in mortality rates reduced inequality in Denmark. Changes in behavioral death causes also reduced inequality (blue bar), while trends in cancer mortality (yellow bar) and other death causes (green bar) both contributed with negligible increases in life expectancy inequality.

If the decline in cardiovascular mortality favored the poor, what caused overall inequality to increase? The answer is differences in survivability due to behavioral causes of death (the middle

\footnotetext{
${ }^{11}$ We base our definition of behavioral diseases on the causes of death studied in Figure 2 of Case and Deaton (2015), which includes a number of diseases often referred to as "deaths of despair" (Case and Deaton, 2017; Ruhm, 2018; Haan et al., 2019). However, we use a slightly broader definition which includes chronic obstructive pulmonary disease. We obtain similar results in an alternative analysis where we only use the diseases from Case and Deaton (2015); see Appendix Section 7.
} 
blue bar). The poor were more likely to die of behavioral death causes before benefiting from the reduction in cardiovascular mortality (ex ante survivability), and those surviving to benefit had fewer remaining life years because of higher mortality from behavioral causes of death in subsequent ages (ex post survivability). Cardiovascular mortality and other causes contribute to the survivability gap between the rich and poor, but their quantitative importance is small compared to behavioral causes.

The third set of cause-specific bars in Figure 5 shows the total change in life expectancy inequality between the rich and poor. Behavioral causes of death are the biggest contributors to the rising gap. This is not because of increasing gaps in behavioral mortality rates, but because initial differences in survivability from behavioral mortality meant that the overall drop in cardiovascular mortality had a smaller impact on life expectancy for the poor than the rich. It is also worth noting that cancer mortality was close to distributionally neutral, both in terms of mortality changes and survivability.

Turning to females, a similar pattern emerges. While cardiovascular mortality dropped more for the poor than the rich (the first red bar for females), higher initial mortality and thus lower survivability for the poor offsets this favorable mortality development. Behavioral causes account for more than half of the contribution from differences in survivability, with cardiovascular and other causes accounting for the rest.

While we cannot link cause of death to income data for the US, as we are able to do for Denmark, we can link cause of death to education level in both the US and Denmark. To account for changes in the composition of education groups over time, we hold the share of the population in each education group fixed. This is similar to using ranks (Meara et al., 2008; Bound et al., 2015). As Novosad et al. (2020) point out, using education as a proxy for social status is not ideal, since education groupings are coarse and the distribution of educational attainment changes over 
time. With these caveats in mind, using high versus low education to define groups, we obtain similar patterns for the two countries, although the magnitudes differ somewhat (see Figure 6). For both countries and both sexes, the drop in mortality rates related to cardiovascular disease helped reduce the gap in life expectancy between the low and high-educated. Nevertheless, total inequality in life expectancy increased. A key reason is the difference between the low and high-educated in survivability in both countries where differences in behavioral causes of death play a major role.

These results show that widening inequality in Denmark is not happening because behavioral mortality differentially worsened for the poor, but because reductions in cardiovascular mortality made existing differences in survivability linked to behavioral mortality more consequential. In the US, changes in behavioral mortality contributed to rising inequality, but a large part of the rising inequality is driven by differences in survivability due to behavioral mortality interacting with the drop in cardiovascular mortality.

The cause-specific decompositions illustrate another important point. Causes of death that contribute most to rising mortality inequality are not necessarily the most important contributors to rising life expectancy inequality. For example, for US males in Figure 6, the increasing inequality in mortality rates is mostly driven by cancers and other causes. Meanwhile, most of the rise in inequality is driven by differences in survivability due to behavioral death causes.

\section{Extensions and Robustness Checks}

In this section, we report a number of additional results supporting our findings. Details are provided in the Appendix.

In the main analysis, we focused on absolute changes in mortality rates as is mostly done in the literature (Currie and Schwandt, 2016; Case and Deaton, 2015; Kinge et al., 2019). One might ask whether differential relative changes in mortality rates between rich and poor would map one-to-one into differential changes in their life expectancy. This turns out not to be the case. Differences in 
survivability are still important and life expectancy may rise more for the rich than the poor, both in absolute terms and in relative terms, even when relative changes in mortality rates are the same for the two groups.

Life expectancy estimates do not account for quality of life. A quantitative approach to address this is to compute disability-adjusted life expectancy, which puts lower weight on life years with a high expected burden of disability (Marmot et al., 2010). When doing this, based on World Health Organization (WHO) estimates of years lived with disability, the rise in inequality becomes smaller. More importantly, the relative role played by differences in survivability between rich and poor in explaining the rise in life expectancy inequality is unchanged.

Recent work suggests it is important to account for income mobility when computing inequality in life expectancy and that this reduces the rise in inequality (Kreiner et al., 2018). This also applies in our case. However, the relative contribution of survivability to the rise in life expectancy inequality is unchanged.

Following recent work on measuring inequality in life expectancy, we proxy social status by position in the income distribution (Chetty et al., 2016; Kreiner et al., 2018; Kinge et al., 2019). As described in Section 5, the same general conclusions apply if we instead use educational attainment. In the appendix, we also provide a decomposition of the trend in life expectancy inequality for nine Western European countries where education data is available. The decomposition results for these countries are broadly similar to the findings for Denmark, suggesting that the results for Denmark are representative of Western European countries.

Finally, to better understand the implications of our decomposition, we perform two counterfactual analyses. We first ask the question: How much would mortality rates of the poor need to decline, keeping the mortality changes of the rich at their actual levels, in order to keep life expectancy inequality unchanged? A common decline in mortality increases life expectancy inequality 
through the survivability effect, so this question asks how much more mortality of the poor would need to decline to keep life expectancy inequality fixed. The answer to this question is contained in Appendix Figure A.8. The blue curves show the actual decline in the mortality rates of the poor at each age. The green curves show how much the mortality rates of the poor would have needed to decline to keep life expectancy inequality constant. For example, for males, age 75 in Denmark, actual mortality fell by around two percentage points, but it would have needed to fall by four percentage points to keep life expectancy inequality unchanged. The graphs for both countries and genders also reveal that it is mortality at higher ages, where most of the deaths are concentrated, which would need to change the most.

Next, we ask which causes of death affect life expectancy inequality most for a given change in mortality. A common drop in mortality rates always increases inequality, since survivability favors the rich at every age. But the increase is larger for mortality reductions occurring at younger ages, since that is when survivability differences are largest (see Figure 2). Therefore, common improvements in causes of death which are more likely among the young (e.g., behavioral causes), lead to larger increases in inequality. Appendix Figure A.9 provides an empirical example based on a common reduction in mortality of 1 percentage point. If this reduction occurs through an improvement in cardiovascular disease, inequality in life expectancy increases by 0.03 years, while the same reduction in behavioral mortality increases inequality by 0.04 years. Thus, a common reduction of mortality increases inequality most if it happens through behavioral death causes (terms 2 and 3 in equation (5)). This effect should be balanced against the possibility of larger declines in mortality of the poor (term 1 in equation (5)). A policy initiative targeting behavioral death causes may well be more favorable in terms of inequality if it reduces mortality more of the poor than the rich compared to an initiative that, say, reduces cardiovascular death to the same extent for rich and poor. 


\section{Conclusion}

Trends in age-specific mortality rates are informative about changes in underlying health status, while trends in life expectancy are a relevant measure of the associated welfare effects. Our decomposition makes clear that focusing on either measure alone can lead to misleading conclusions. In Denmark, inequality in age-specific mortality rates decreased, while inequality in life expectancy increased. Studying the increasing gap in life expectancy in isolation could lead to the erroneous conclusion that factors such as medical innovations or changes in behavior favored the rich. Likewise, studying the decreasing gap in mortality in isolation would lead to the erroneous conclusion that the gap in welfare between rich and poor was closing.

Survivability ties together these seemingly contradictory findings for Denmark. The larger reductions in mortality rates of the poor led to smaller gains in life expectancy because more rich than poor lived to benefit (ex ante survivability) and the poor gained fewer remaining life years if

they did live to benefit (ex post survivability). In the US, inequality in mortality rates increased, and this was amplified by differences in survivability, implying an even larger increase in life expectancy inequality. 


\section{References}

Arriaga, E. E. (1984). Measuring and explaining the change in life expectancies. Demography 21(1), $83-96$.

Attanasio, O. P. and T. H. Nielsen (2020). Economic Resources, Mortality and Inequality. CEBI Working Paper 06/20, University of Copenhagen.

Auerbach, A. J., K. K. Charles, C. C. Coile, W. Gale, D. Goldman, R. Lee, C. M. Lucas, P. R. Orszag, L. M. Sheiner, B. Tysinger, D. N. Weil, J. Wolfers, and R. Wong (2017). How the Growing Gap in Life Expectancy May Affect Retirement Benefits and Reforms. Geneva Papers on Risk and Insurance: Issues and Practice 42(3), 475-499.

Baker, M., J. Currie, and H. Schwandt (2019). Mortality inequality in Canada and the United States: Divergent or convergent trends? Journal of Labor Economics 37(S2), S325-S353.

Blinder, A. S. (1973). Wage Discrimination: Reduced Form and Structural Estimates. The Journal of Human Resources 8(4), 436-455.

Bor, J., G. H. Cohen, and S. Galea (2017). Population health in an era of rising income inequality: USA, 1980-2015. Lancet 389(10077), 1475-1490.

Bound, J., A. T. Geronimus, J. M. Rodriguez, and T. A. Waidmann (2015). Measuring recent apparent declines in longevity: The role of increasing educational attainment. Health Affairs.

Canudas Romo, V. (2003). Decomposition Methods in Demography. Max Planck Institute for Demographic Research (June), 1-178.

Case, A. and A. Deaton (2015). Rising morbidity and mortality in midlife among white non-Hispanic Americans in the 21st century. Proceedings of the National Academy of Sciences of the United States of America 112(49), 15078-15083.

Case, A. and A. Deaton (2017). Mortality and morbidity in the 21st century. BPEA Conference Drafts, 1-60.

Chetty, R., M. Stepner, S. Abraham, S. Lin, B. Scuderi, N. Turner, A. Bergeron, and D. Cutler (2016). The association between income and life expectancy in the United States, 2001-2014. JAMA - Journal of the American Medical Association 315(16), 1750-1766.

Currie, J. and H. Schwandt (2016). Inequality in mortality decreased among the young while increasing for older adults, 1990-2010. Science 352(6286), 708-712.

Cutler, D., A. Deaton, and A. Lleras-Muney (2006). The determinants of mortality. Journal of Economic Perspectives 20(3), 97-120.

Cutler, D. and S. Kadiyala (2003). Chapter 4: The Return to Biomedical Research: Treatment and Behavioral Effects. In Measuring the gains from medical research: An economic approach. Chicago and London: University of Chicago Press.

Cutler, D. M., F. Lange, E. Meara, S. Richards-Shubik, and C. J. Ruhm (2011). Rising educational gradients in mortality: The role of behavioral risk factors. Journal of Health Economics 30(6), $1174-1187$.

Deaton, A. (2013). The Great Escape - Health, Wealth, and the Origins of Inequality. Princeton University Press.

Fortin, N., T. Lemieux, and S. Firpo (2011). Decomposition Methods in Economics. In Handbook of Labor Economics, Volume 4, pp. 1-102.

Goldin, C. and A. Lleras-Muney (2019). XX $>$ XY?: The changing female advantage in life expectancy. Journal of Health Economics 67.

Guillot, M. (2011). Chapter 25 Period Versus Cohort Life Expectancy. In International Handbook of Adult Mortality, pp. 533-549. 
Haan, P., A. Hammerschmid, and J. Schmieder (2019). Mortality in midlife for subgroups in Germany. Journal of the Economics of Ageing 14.

Hederos, K., M. Jäntti, L. Lindahl, and J. Torssander (2017). Trends in Life Expectancy by Income and the Role of Specific Causes of Death. Economica 85, 606-625.

Ho, J. Y. and A. S. Hendi (2018). Recent trends in life expectancy across high income countries: Retrospective observational study. BMJ (Online) 362.

Honoré, B. E. and A. Lleras-Muney (2006). Bounds in competing risks models and the war on cancer. Econometrica 74 (6), 1675-1698.

Jayachandran, S., A. Lleras-Muney, and K. V. Smith (2010). Modern medicine and the twentieth century decline in mortality: Evidence on the impact of sulfa drugs. American Economic Journal: Applied Economics 2(2), 118-146.

Kinge, J. M., J. H. Modalsli, S. Øverland, H. K. Gjessing, M. C. Tollånes, A. K. Knudsen, V. Skirbekk, B. H. Strand, S. E. Håberg, and S. E. Vollset (2019). Association of Household Income with Life Expectancy and Cause-Specific Mortality in Norway, 2005-2015. JAMA - Journal of the American Medical Association 321(19), 1916-1925.

Kitagawa, E. M. (1955). Components of a Difference Between Two Rates. Journal of the American Statistical Association 50(272), 1168-1194.

Kreiner, C. T., T. H. Nielsen, and B. L. Serena (2018). Role of income mobility for the measurement of inequality in life expectancy. Proceedings of the National Academy of Sciences of the United States of America 115(46), 11754-11759.

Likosky, D. S., J. Van Parys, W. Zhou, W. B. Borden, M. C. Weinstein, and J. S. Skinner (2018). Association between medicare expenditure growth and mortality rates in patients with acute myocardial infarction: A comparison from 1999 through 2014. JAMA Cardiology 3(2), 114-122.

Lleras-Muney, A. (2005). The relationship between education and adult mortality in the United States. Review of Economic Studies 72(1), 189-221.

Mackenbach, J. P., J. R. Valverde, B. Artnik, M. Bopp, H. Brønnum-Hansen, P. Deboosere, R. Kalediene, K. Kovács, M. Leinsalu, P. Martikainen, G. Menvielle, E. Regidor, J. Rychtaríkova, M. Rodriguez-Sanz, P. Vineis, C. White, B. Wojtyniak, Y. Hu, and W. J. Nusselder (2018). Trends in health inequalities in 27 European countries. Proceedings of the National Academy of Sciences of the United States of America 115(25), 6440-6445.

Marmot, M., J. Allen, P. Goldblatt, T. Boyce, D. McNeish, M. Grady, and I. Geddes (2010). Fair society, healthy lives (The Marmot Review): Strategic Review of Health Inequalities in English post-2010. Technical report.

Meara, E. R., S. Richards, and D. M. Cutler (2008). The gap gets bigger: Changes in mortality and life expectancy, by education, 1981-2000. Health Affairs 27(2), 350-360.

Montez, J. K., A. Zajacova, M. D. Hayward, S. H. Woolf, D. Chapman, and J. Beckfield (2019). Educational Disparities in Adult Mortality Across U.S. States: How Do They Differ, and Have They Changed Since the Mid-1980s? Demography 56(2), 621-644.

Moscelli, G., L. Siciliani, N. Gutacker, and R. Cookson (2018). Socioeconomic inequality of access to healthcare: Does choice explain the gradient? Journal of Health Economics 57, 290-314.

Novosad, P., C. Rafkin, and S. Asher (2020). Rising Mortality Among Less Educated Americans. Unpublished Manuscript.

Oaxaca, R. (1973). Male-Female Wage Differentials in Urban Labor Markets. International Economic Review 14(3), 693-709.

Poterba, J. M. (2014). Retirement security in an aging population. American Economic Review $104(5), 1-30$. 
Ruhm, C. J. (2018). Drug Mortality and Lost Life Years Among U.S. Midlife Adults, 1999-2015. American Journal of Preventive Medicine 55(1), 11-18.

Snyder, S. E. and W. N. Evans (2006). The Effect of Income on Mortality : Evidence from the Social Security Notch. Review of Economics and Statistics 88(3), 482-495.

van den Berg, G. J., U. G. Gerdtham, S. von Hinke, M. Lindeboom, J. Lissdaniels, J. Sundquist, and K. Sundquist (2017). Mortality and the business cycle: Evidence from individual and aggregated data. Journal of Health Economics 56, 61-70.

Waldron, H. (2007). Trends in mortality differentials and life expectancy for male social securitycovered workers, by socioeconomic status. Social Security Bulletin 67(3), 1-28. 


\section{Tables}

Table 1: Effect of mortality change at age 60 on life expectancy of 40-year-old males

\begin{tabular}{lcccc}
\hline$\Delta$ Mortality & Survival & Remain. LE & Survivability & $\Delta$ Life Exp \\
$\Delta M_{60}$ & $S_{60}$ & $R_{60}$ & $X_{60}$ & $\Delta L E_{40}$ \\
$(1)$ & $(2)$ & $(3)$ & $(4)=(2) \times(3)$ & $(5)=(1) \times(4)$ \\
\hline United States & & & & \\
Poor -0.002 & 0.84 & 19.0 & 16.0 & 0.032 \\
Rich -0.002 & 0.95 & 23.5 & 22.3 & 0.045 \\
Denmark & & & & \\
Poor -0.003 & 0.78 & 15.6 & 12.2 & 0.037 \\
Rich -0.003 & 0.94 & 19.6 & 18.4 & 0.055 \\
\hline
\end{tabular}

Notes: The table shows the effect of the observed same change in the mortality rate of rich and poor 60 year old males on life expectancy at age 40. The table reports: (1) changes in mortality rates during 2001-2014, (2) survival probabilities, $S_{60}$, up to age $60,(3)$ remaining life expectancy after age $60, R_{60}$, (4) survivability at age 60 , $X_{60} \equiv S_{60} \cdot R_{60}$, and (5) the effect on life expectancy at age 40 from the changes in mortality rates at age 60 . The change in life expectancy in column (5) is computed from the first-order approximation in equation (2). Survival probabilities and remaining life expectancies are calculated using the definitions reported below equation (2) and measured in 2001 in accordance with the first-order approximation. To match the numbers in Figure 2, which plots mortality rate changes and survivability by 5 -year age bins, all numbers are calculated as the average of individuals aged 60-64 years. 


\section{Figures}

Figure 1: Life expectancy at age 40 by income class, US and Denmark

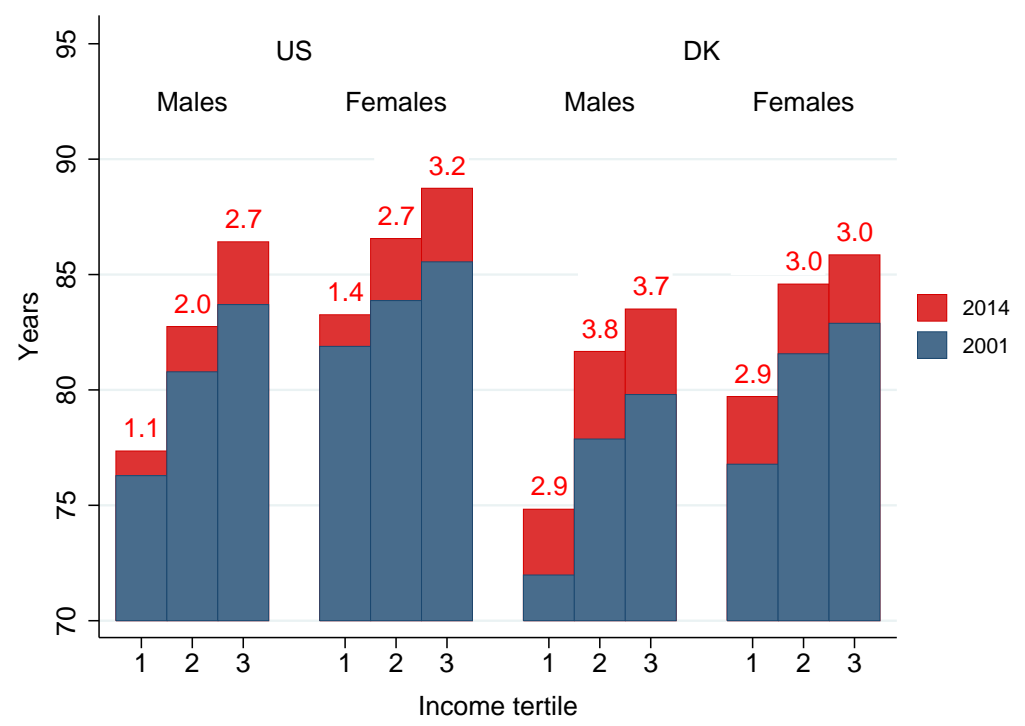

Notes: The figure shows the average life expectancy at age 40 in the US and Denmark by income tertiles in 2001 and 2014 for males and females. The numbers above the bars denote the increase from 2001 to 2014. The figure is constructed by computing period life expectancy for each tertile group using equation (1) and data on mortality rates by age and income class. The mortality rates for the US are from Chetty et al. (2016) and are based on all individuals who file tax returns. The mortality rates for Denmark are computed by the authors and cover the entire population. The data is described in greater detail in Section 3. 
Figure 2: Mortality changes and survivability, by age and income class

A. Mortality rate change
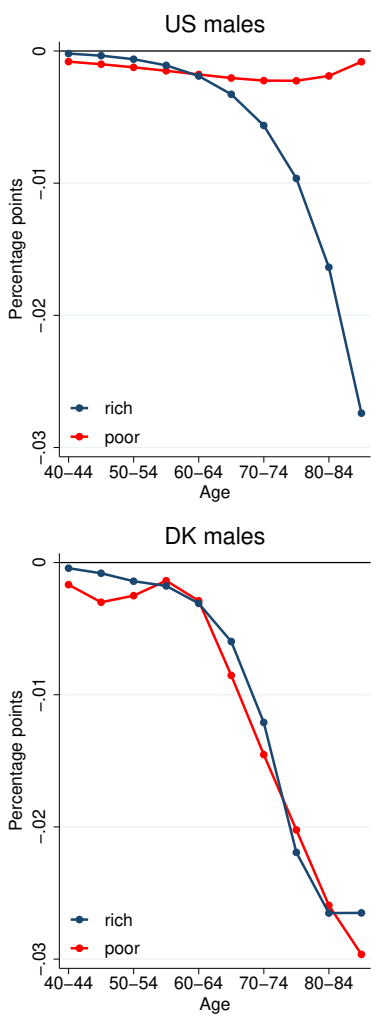
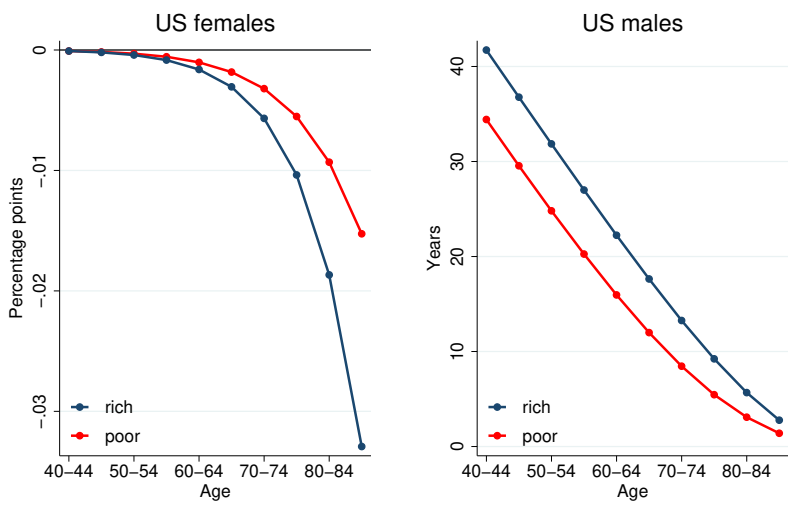

B. Survivability
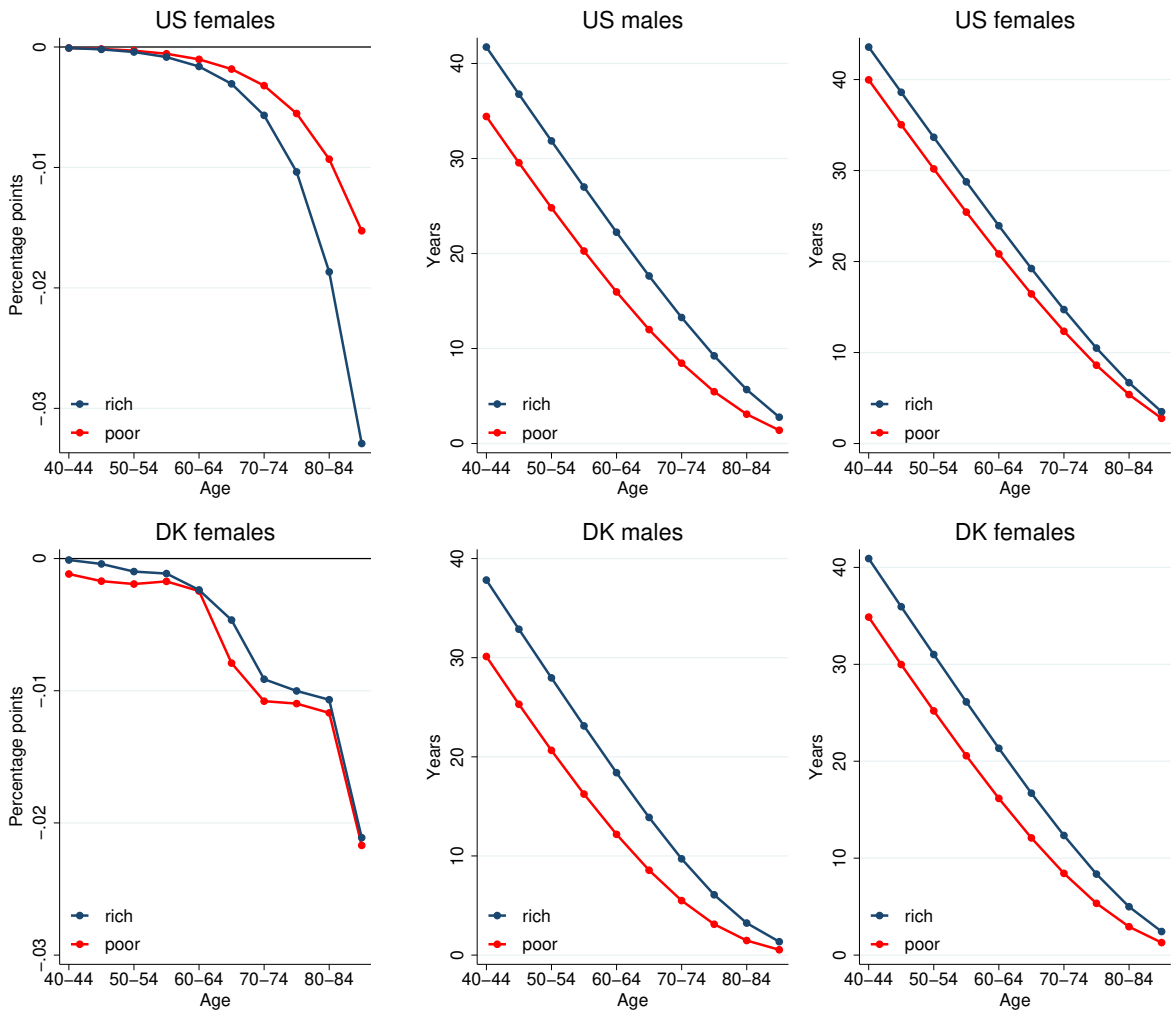

Notes: Panel A plots changes in mortality rates from 2001 to 2014 by 5 -year age bins for poor (tertile 1) and rich (tertile 3) males and females in the US and Denmark. The figures are constructed by computing average changes in mortality rates within 5-year age groups. Panel B plots survivability by 5-year age bins for the same groups. These figures are constructed by first using the definition in equation (2) and data on mortality rates to compute survivability, $X_{a}$, in 2001 and then averaging across 5-year age groups. 
Figure 3: Change in life expectancy gap between rich and poor at age 40 decomposed into differential mortality rate changes and differences in survivability.

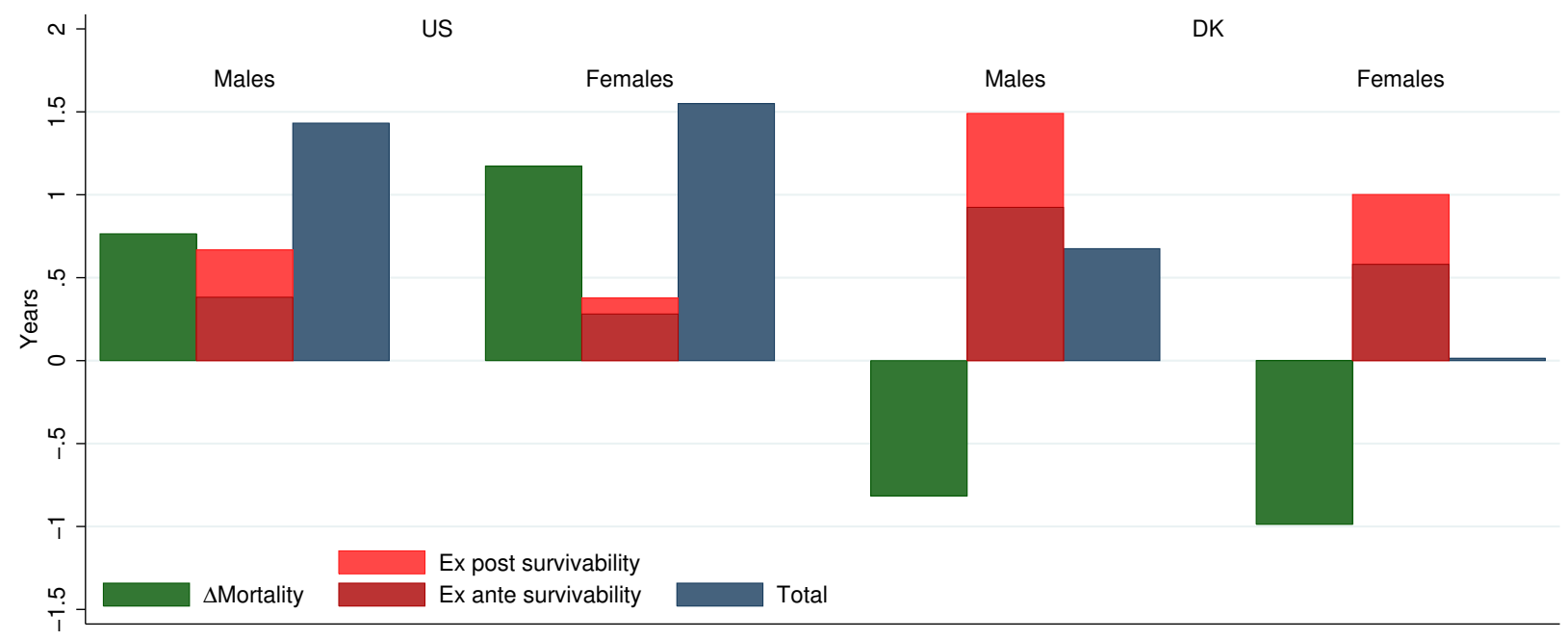

Notes: To create the figure, we use the decomposition formulas in equations (3) and (4) and the data on mortality rates from the US and Denmark to compute the change in life expectancy inequality between the rich and poor during 2001-2014 (shown in blue bars) and to decompose this into contributions from differential changes in mortality rates (green bars) and differences in (ex ante and ex post) survivability (red bars). 
Figure 4: Trends in mortality for individuals aged 40-100 for the US and Denmark split by four causes of death: cardiovascular, behavioral, cancers, and other diseases.

A. US

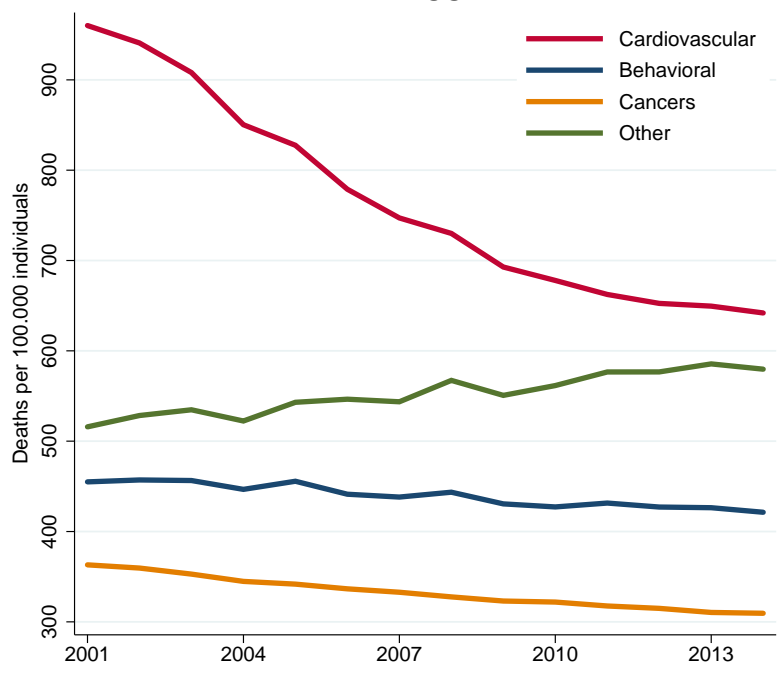

A. DK

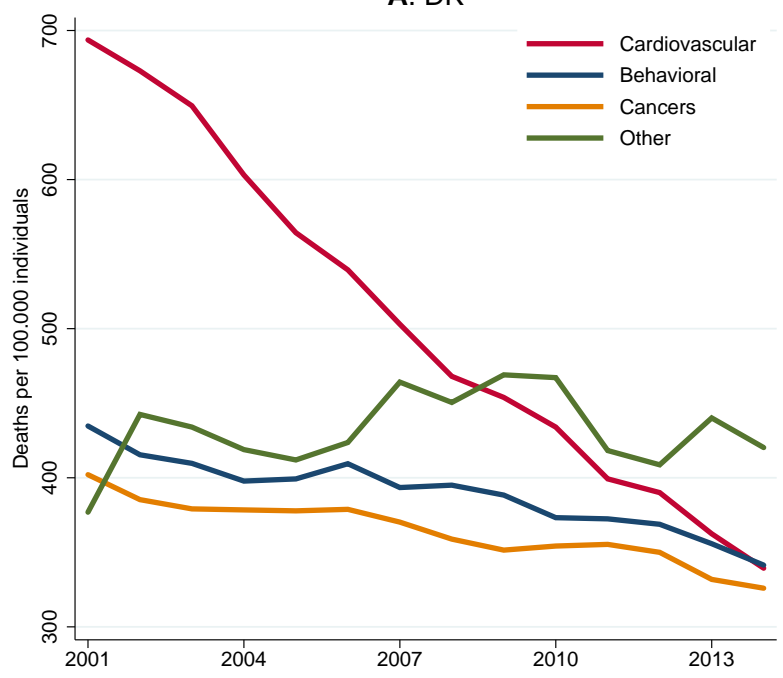

Notes: The figure plots age-standardized mortality per 100,000 individuals by cause of death for the years 2001 to 2014 for the US (Panel A) and Denmark (Panel B). US mortality rates are calculated from WHO population estimates (http://ghdx.healthdata.org/gbd-results-tool) and mortality by cause of death (http://apps.who.int/ healthinfo/statistics/mortality/causeofdeath_query/start.php). The age standardization uses the US standard population from the World Health Organization, downloaded from https://seer.cancer.gov/stdpopulations/ stdpop. singleages.html. 
Figure 5: Cause-specific decomposition of change in life expectancy inequality, DK

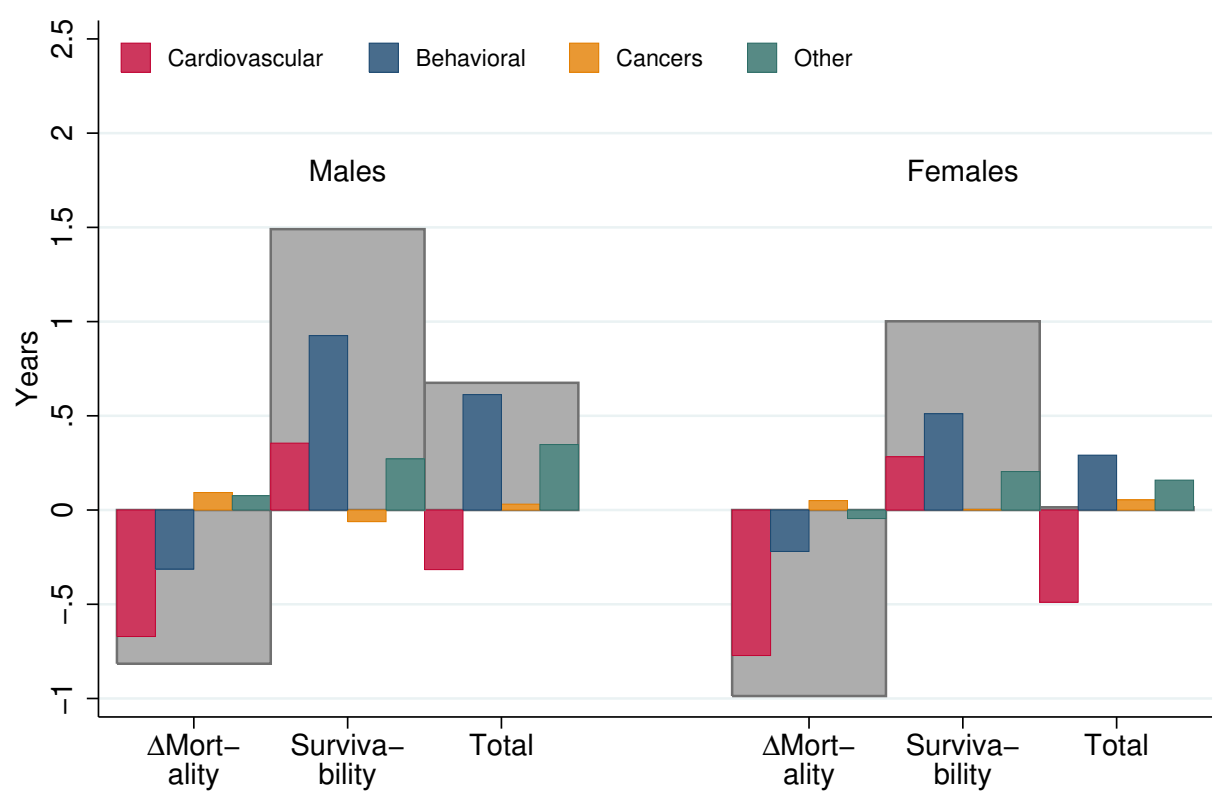

Notes: The figure shows the cause-specific decomposition of changes in inequality in life expectancy measured at age 40 and computed using equation (5) based on cause and income-specific mortality rates in the Danish data.

Figure 6: Cause-specific decomposition of change in life expectancy inequality by education

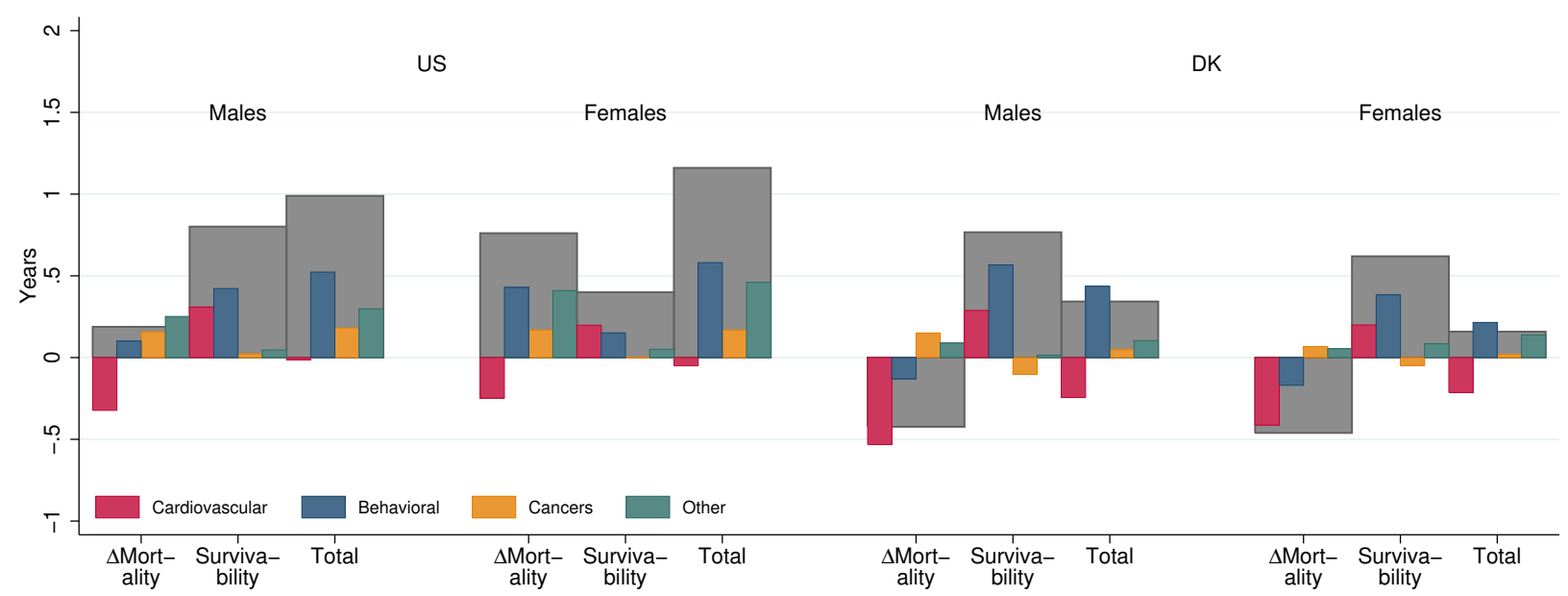

Notes: The figure shows cause-specific decompositions of changes in inequality in life expectancy in the US and Denmark measured at age 40 and computed using equation (5) based on cause and education-specific mortality rates. For the US results, we use data from Case and Deaton (2017) and consider inequality between individuals with high school or less vs. BA or more. For the Danish results, we define low and high education based on the International Standard Classification of Education 1997 (ISCED) codes; low education: 0-2, high education: 5+. 


\section{Online Appendix for \\ Linking Changes in Inequality in Life Expectancy and Mortality: Evidence from Denmark and the United States}

Gordon B. Dahl, Claus Thustrup Kreiner, Torben Heien Nielsen, Benjamin Ly Serena

\section{Contents}

1 Data

2 Comparability of results for the US and Denmark $\quad 5$

3 Derivation of decomposition formulas $\quad 6$

4 Accuracy of first order approximation $\quad 8$

5 Derivation of cause-specific decomposition $\quad 9$

6 Alternative definition of behavioral diseases $\quad 11$

7 Relative changes in mortality rates and life expectancy 11

8 Accounting for income mobility $\quad 13$

9 Disability-adjusted life expectancy $\quad 14$

10 Inequality trends in other Western European countries $\quad 16$

11 Appendix figures and tables $\quad 19$ 


\section{Data}

US

We use publicly available data from Chetty et al. (2016) on income-specific mortality in the US during the years 2001-2014. The income-specific mortality rates are estimated by Chetty et al. (2016) using administrative tax data covering the entire US population. To minimize reverse causality, mortality rates are estimated based on income measured two years prior. The income concept is defined as the sum of household gross income minus Social Security and disability benefits. For individuals who do not file taxes, the income concept includes wage earnings and unemployment benefits. The authors exclude observations with zero income. This restriction drops about 9 percent of the population. As a large fraction of individuals with zero income are disability insurance recipients and these have above-normal rates of mortality, the computed life expectancies are overstated compared to the population averages in the US. After the public retirement age earnings become a poor measure of socioeconomic status. The authors therefore impute mortality rates using Gompertz approximations, which assumes mortality is log-linear in age, $\log \left(M_{a}\right)=\alpha+\beta *$ Age (see Chetty et al., 2016). After age 90, at which point the Gompertz approximation is less accurate, the authors replace age and income-specific mortality rates with age-specific, population-wide mortality rates. In addition, Chetty et al. (2016) adjusts mortality rates for differences in the composition of ethnicity across income groups.

\section{Denmark}

We use Danish administrative data provided by Statistics Denmark and covering the period 20012014 for the universe of Danish residents. We link the population registers, BEF and FAIN, containing information on sex and age, with the income register, IND, and the death registers, DODAARS and DODAASG, using personal identifiers (CPR number). Following previous work (Kreiner et al., 
2018), we measure income as household income net of universal transfers:

$$
\text { Income }=\text { PERINDKIALT }- \text { QPENSNY }- \text { KONTHJ }- \text { ANDOVERFORSEL }
$$

PERINDKIALT is total income, QPENSNY is public pensions (disability pension, public retirement pension), KONTHJ is cash assistance, and ANDOVERFORSEL is other universal public transfers. Household income is defined as the mean income of the individual and the spouse (if the individual of interest is either married or cohabiting). Following the previous literature (Chetty et al., 2016; Kreiner et al., 2018), we measure income two years before we measure mortality to reduce the importance of reverse causality. Because the data covers the entire population of Denmark, the estimated life expectancies line up closely with official estimates from Statistics Denmark. The income measure we use includes payouts from private and employer-based pension accounts, which are tightly linked to previous labor market earnings. For this reason, our income concept remains a good measure of socioeconomic status after retirement. See Kreiner et al. (2018) for a validation of the income measure and the stability of income ranks around retirement.

Below, in the section titled 'Comparability of results for US and Denmark', we discuss how differences in data sources affect comparisons between Denmark and the US and present estimates for Denmark based on data restrictions similar to those in the US data.

\section{Education Data}

We use education attainment as an alternative measure of socioeconomic status. For the US, we replicate estimates of mortality by education in the US by Case and Deaton (2017) using data from the National Vital Statistics and the March Current Population Survey. As in Case and Deaton (2017), we consider the difference in life expectancy between individuals with high school or less, some college, and BA or more. In Denmark, where data on highest completed education is available from administrative records, we follow Mackenbach et al. (2018) and define low, middle, and high 
education groups using International Standard Classification of Education 1997 (ISCED) codes; low: $0-2$, middle: $3-4$, high: $5+$. To account for changes in the composition of education groups over time, we hold the share of the population in each education group fixed. Hence, as the share of the population in low education groups decreases over time, we randomly allocate some individuals in the middle education group to the low education group. This is similar to using ranks, as we do for income.

In both Denmark and the US, education information is only available for individuals below age 80. Therefore, we impute mortality from age 80 to 100 using Gompertz approximations. Following Chetty et al. (2016), we replace these approximations with population-wide mortality rates after age 90 , because the Gompertz approximation is less accurate after this age.

The mortality and education data from Case and Deaton (2017) contain information on causespecific mortality for the US, allowing us to conduct cause-specific decompositions. However, because we impute mortality rates by education after age 80 , information on cause of death by education is not available in these ages. To deal with this issue, we assume that the relative importance of different diseases for total mortality across education groups is fixed at the age 80 level. Hence, if the share of deaths from cardiovascular disease within an education group is twice as large for the low-educated as the high-educated at age 80 , we assume that this is also the case at age 81 and so forth. ${ }^{1}$ After age 90, all education groups are assigned population-wide, cause-specific mortality rates.

\footnotetext{
${ }^{1}$ Assume that the share of deaths due to a given disease is proportional across education groups $(l o w=l, m i d=m$, high $=\mathrm{h})$ :

$$
\frac{D_{a, c}^{l}}{D_{a}^{l}}=x \cdot \frac{D_{a, c}^{m}}{D_{a}^{m}}, \quad \frac{D_{a, c}^{l}}{D_{a}^{l}}=y \cdot \frac{D_{a, c}^{h}}{D_{a}^{h}}
$$

where $D_{a, c}$ is cause-specific mortality and the values of $x$ and $y$ are measured at age 80 and assumed constant thereafter. The sum of education-specific mortality rates must sum to total mortality:

$$
N_{a}^{l} \cdot D_{a, c}^{l}+N_{a}^{m} \cdot D_{a, c}^{m}+N_{a}^{h} \cdot D_{a, c}^{h}=D_{a, c}
$$

where $N_{a}^{e}$ denotes the share of individuals alive at age $a$ that belong to education group $e$. To derive cause-specific mortality rates by education, $D_{a, c}^{e}$, isolate $D_{a, c}^{m}$ and $D_{a, c}^{h}$ from equation (A.2) and insert in equation (A.3). Simplify and isolate $D_{a, c}^{l}$ :
} 


\section{Construction of dataset for the main analysis}

The main results are based on age-specific mortality rates by income tertiles in 2001 and 2014 . This is constructed by first computing annual mortality rates by age, sex, year, and income tertile (within cohort, sex, and year) for the ages 40-100 and years 2001-2014. To maximize precision, we then use all years during 2001-2014 to run regressions of age-specific mortality rates on linear trends and use the predicted age-specific mortality rates in 2001 and 2014 rather than the actual mortality rates for these two years. For Denmark, we have information on causes of death. Therefore, we also compute four different cause-specific mortality rates; cardiovascular (ICD-10 codes: I00-I78), behavioral (ICD-10 codes: C32-C34, E10-E14, F10-F19, J20-J22, J40-J47, K70-K83, V01-V99, W00-W99, X00-X97, Y00-Y89), cancers (ICD-codes: C00-C31, C37-C97, D00-D09), and other (all remaining ICD-10 codes). We follow the same procedure as for all-cause mortality rates and predict cause-specific mortality rates in 2001 and 2014 using regressions with linear trends through all years during 2001-2014.

\section{Comparability of results for the US and Denmark}

The US income data differ in important ways from the Danish data. While the Danish data covers the entire population, the US data does not include individuals with zero income, many of whom are disability insurance recipients. This sample restriction drops $9 \%$ of the US population and $32 \%$ of deaths. Therefore, mortality rates for the US are underestimated and life expectancies are overestimated compared to population averages. In Figure A.1, we exclude individuals with zero or negative income and disability insurance recipients from the Danish data, to make results for

$$
D_{a, c}^{l}=\frac{D_{a, c}}{\frac{D_{a}^{m} \cdot N_{a}^{m}}{D_{a}^{l} \cdot x}+N_{a}^{l}+\frac{D_{a}^{h} \cdot N_{a}^{h}}{D_{a}^{l} \cdot y}}
$$

$D_{a, c}^{m}$ and $D_{a, c}^{h}$ then follow from equation (A.2). This imputation does not ensure that the sum of the cause-specific mortality rates sum to aggregate mortality within each education group. However, it is very close empirically. We fix the small difference by multiplying cause and education-specific mortality rates with $\frac{D_{a}^{e}}{\sum_{c} D_{a, c}^{e}}$. 
Denmark and the US more comparable. These restrictions drop 9\% of the Danish population and $36 \%$ of deaths. After the public retirement age, disability insurance recipients receive a public pension and cannot be identified in the data. Instead, we calculate the share of individuals in each income group dropped by our restrictions before retirement (age 60) and drop the same share in post retirement ages.

Figure A.1A plots the average life expectancy in Denmark and the US by income tertiles in 2001 and 2014 for males and females. As most of the disability insurance recipients have high mortality and low income, implementing the US sample restrictions increases average life expectancy and reduces inequality in Denmark. For example, the difference between males in the bottom and top tertile of income in 2001 is 5 years rather than 8 years. As shown in Figure A.1B, the rise in life expectancy inequality among Danish males from 2001 to 2014 is also smaller; 0.5 years instead of 0.7 years. However, the sample restriction does not change our main conclusions. Declines in mortality over time are larger for the poor than the rich, and the rise in life expectancy inequality in Denmark is driven entirely by differences in survivability.

\section{Derivation of decomposition formulas}

Equation (2) in the main text is derived by differentiation of the definition for life expectancy in equation (1) in the main text. By differentiating this expression with respect to the mortality rate $M_{a}$ at age $a$, we get the first-order approximation:

$$
\begin{aligned}
\Delta L E_{\underline{a}} & \approx-\Delta M_{a} \cdot \prod_{i=\underline{a}}^{a-1}\left(1-M_{i}\right) \cdot \sum_{j=a}^{\bar{a}} \prod_{i=a+1}^{j}\left(1-M_{i}\right) \\
& \approx-\Delta M_{a} \cdot \prod_{i=\underline{a}}^{a-1}\left(1-M_{i}\right) \cdot\left(1+\sum_{j=a+1}^{\bar{a}} \prod_{i=a+1}^{j}\left(1-M_{i}\right)\right)
\end{aligned}
$$


where we have used the definition $\prod_{i=a+1}^{a}\left(1-M_{i}\right) \equiv 1$. Next, we define

$$
S_{a} \equiv \prod_{i=\underline{a}}^{a-1}\left(1-M_{i}\right), \quad R_{a} \equiv 1+\sum_{j=a+1}^{\bar{a}} \prod_{i=a+1}^{j}\left(1-M_{i}\right)
$$

where $S_{a}$ is the probability of survival from age $\underline{a}$ to age $a$ and $R_{a}$ is remaining life expectancy if surviving age $a$, in which case the individual lives one extra year for sure and from age $a+1$ can expect to live the additional years given by the last term in the definition. This implies that the effect of a change in the mortality rate $M_{a}$ at age $a$ may be written as:

$$
\Delta L E_{\underline{a}} \approx-\Delta M_{a} \cdot X_{a} \text { where } X_{a} \equiv S_{a} \cdot R_{a}
$$

The effect of a change in all mortality rates $\left\{M_{i}\right\}_{\underline{a}}^{\bar{a}}$ can be found by total differentiation of definition (1) in the main text, which corresponds to a simple aggregation of equation (A.5). This gives

$$
\Delta L E_{\underline{a}} \approx-\sum_{a=\underline{a}}^{\bar{a}} \Delta M_{a} \cdot X_{a}
$$

This is equation (2) in the main text. We proceed by deriving changes in life expectancy inequality over time. We study life expectancy inequality between a poor group $\left(L E^{p}\right)$ and a rich group $\left(L E^{r}\right)$ with equally many individuals in each group (bottom one-third versus top one-third). Using equation (A.6), the change in life expectancy inequality over time equals:

$$
\Delta L E_{\underline{a}}^{r}-\Delta L E_{\underline{a}}^{p} \approx-\sum_{a=\underline{a}}^{\bar{a}}\left(\Delta M_{a}^{r} \cdot X_{a}^{r}-\Delta M_{a}^{p} \cdot X_{a}^{p}\right) .
$$

Equation (A.6) may be rewritten as

$$
\begin{aligned}
\Delta L E_{\underline{a}}^{k} & \approx-\sum_{a=\underline{a}}^{\bar{a}} \Delta M_{a}^{k} \cdot \overline{X_{a}}-\sum_{a=\underline{a}}^{\bar{a}} \overline{\Delta M}_{a} \cdot\left(X_{a}^{k}-\overline{X_{a}}\right) \\
& -\sum_{a=\underline{a}}^{\bar{a}}\left(\Delta M_{a}^{k}-{\overline{\Delta M_{a}}}\right) \cdot\left(X_{a}^{k}-\overline{X_{a}}\right), \quad k=r, p
\end{aligned}
$$


where superscript $k$ denotes the income class. By inserting this expression in equation (A.7), we get:

$$
\begin{aligned}
\Delta L E_{\underline{a}}^{r}-\Delta L E_{\underline{a}}^{p} \approx & -\sum_{a=\underline{a}}^{\bar{a}}\left(\Delta M_{a}^{r}-\Delta M_{a}^{p}\right) \cdot \overline{X_{a}}-\sum_{a=\underline{a}}^{\bar{a}} \overline{\Delta M_{a}} \cdot\left(X_{a}^{r}-X_{a}^{p}\right) \\
& -\sum_{a=\underline{a}}^{\bar{a}}\left(\Delta M_{a}^{r}-\overline{\Delta M}_{a}\right) \cdot\left(X_{a}^{r}-\overline{X_{a}}\right) \\
& +\sum_{a=\underline{a}}^{\bar{a}}\left(\Delta M_{a}^{p}-\overline{\Delta M}_{a}\right) \cdot\left(X_{a}^{p}-\overline{X_{a}}\right)
\end{aligned}
$$

Next, we insert the definition of the averages $\overline{X_{a}}=\frac{1}{2}\left(X_{a}^{r}+X_{a}^{p}\right)$ and $\overline{\Delta M}_{a}=\frac{1}{2}\left(\Delta M_{a}^{r}+\Delta M_{a}^{p}\right)$ :

$$
\begin{aligned}
\Delta L E_{\underline{a}}^{r}-\Delta L E_{\underline{a}}^{p} \approx & -\sum_{a=\underline{a}}^{\bar{a}}\left[\left(\Delta M_{a}^{r}-\Delta M_{a}^{p}\right) \cdot \overline{X_{a}}+\overline{\Delta M}_{a} \cdot\left(X_{a}^{r}-X_{a}^{p}\right)\right] \\
& -\sum_{a=\underline{a}}^{\bar{a}} \frac{1}{4}\left(\Delta M_{a}^{r}-\Delta M_{a}^{p}\right) \cdot\left(X_{a}^{r}-X_{a}^{p}\right) \\
& +\sum_{a=\underline{a}}^{\bar{a}} \frac{1}{4}\left(\Delta M_{a}^{p}-\Delta M_{a}^{r}\right) \cdot\left(X_{a}^{p}-X_{a}^{r}\right) .
\end{aligned}
$$

The last two (covariance) terms cancel out because the two groups are of the same size. Thus, we arrive at

$$
\Delta L E_{\underline{a}}^{r}-\Delta L E_{\underline{a}}^{p} \approx \sum_{a=\underline{a}}^{\bar{a}} \overline{X_{a}} \cdot\left(\Delta M_{a}^{p}-\Delta M_{a}^{r}\right)+\sum_{a=\underline{a}}^{\bar{a}} \overline{\Delta M_{a}} \cdot\left(X_{a}^{p}-X_{a}^{r}\right)
$$

which is equation (3) in the main text.

\section{Accuracy of first order approximation}

The age decomposition formula (2) in the main text is a first-order approximation of the change in life expectancy over time. Figure A.2A shows the difference between the first order approximation and the actual changes in life expectancy for the rich and poor. Overall, the first order approximation provides increases which are too small over time in life expectancy and life expectancy inequality. However, the errors are relatively small. For US females, which has the largest approximation error, 
the actual increase in life expectancy inequality during 2001-2014 is 1.8 years, while the first order approximation gives 1.5 years.

Often researchers use an Arriaga age decomposition (Arriaga, 1984). In addition to the firstorder approximation (2) in the main text, this approximation includes an extra term, an interaction effect, equal to $\Delta R_{a} \cdot \Delta M_{a} \cdot S_{a}$. The interaction effect cannot be attributed to any particular age, but reduces the approximation error of the age decomposition. Figure A.2B shows that our decomposition of trends in life expectancy inequality into differential changes in mortality rates of rich and poor and differences in survivability is more or less unchanged if we include the interaction effect in the age decomposition. Differences in survivability still explain all of the increase in life expectancy inequality in Denmark and between $25-50 \%$ of the increase in the US.

\section{Derivation of cause-specific decomposition}

This section describes how we estimate the cause-specific contributions to the change in life expectancy inequality over time and its decomposition into mortality rate changes and survivability in Section 5 of the paper. We start from equation (4) in the main text, which decomposes changes in life expectancy inequality into contributions from changes in mortality rates, ex ante survivability, and ex post survivability:

$$
\begin{aligned}
\Delta L E_{\underline{a}}^{r}-\Delta L E_{\underline{a}}^{p} \approx & \underbrace{\sum_{a=\underline{a}}^{\bar{a}} \overline{X_{a}} \cdot\left(\Delta M_{a}^{p}-\Delta M_{a}^{r}\right)}_{\Delta \text { Mortality }} \\
& +\underbrace{\sum_{a=\underline{a}}^{\bar{a}} \overline{\Delta M_{a}} \cdot \overline{R_{a}} \cdot\left(S_{a}^{p}-S_{a}^{r}\right)}_{\text {Ex ante survivability }}+\underbrace{\sum_{a=\underline{a}}^{\bar{a}} \overline{\Delta M_{a}} \cdot \overline{S_{a}} \cdot\left(R_{a}^{p}-R_{a}^{r}\right)}_{\text {Ex post survivability }}
\end{aligned}
$$

Next, we decompose each of the three terms separately by causes of death denoted by the index $c$. 


\section{Mortality rate changes by cause of death}

As cause-specific mortality rates sum to total mortality, it is straightforward to decompose mortality rate changes by cause $c$ :

$$
\sum_{a=\underline{a}}^{\bar{a}} \bar{X}_{a} \cdot\left(\Delta M_{a}^{p}-\Delta M_{a}^{r}\right)=\sum_{a=\underline{a}}^{\bar{a}} \bar{X}_{a} \cdot \sum_{c}\left(\Delta M_{a, c}^{p}-\Delta M_{a, c}^{r}\right)
$$

\section{Ex ante survivability by cause of death}

To decompose ex ante survivability by cause of death, we rewrite income differences in the probability of surviving to a certain age $a$, i.e., $S_{a}^{p}-S_{a}^{r}$, as the sum of differences in cause-specific survival probabilities $S_{a}^{p}-S_{a}^{r}=\sum_{c}\left(S_{a, c}^{p}-S_{a, c}^{r}\right)$, where the cause-specific survival probabilities reflect the probability that a person does not die from that particular disease between age $\underline{a}$ and $a$. Inserting this into the expression for ex ante survivability yields:

$$
\sum_{a=\underline{a}}^{\bar{a}} \overline{\Delta M_{a}} \cdot \overline{R_{a}} \cdot\left(S_{a}^{p}-S_{a}^{r}\right)=\sum_{a=\underline{a}}^{\bar{a}} \overline{\Delta M_{a}} \cdot \overline{R_{a}} \cdot \sum_{c}\left(S_{a, c}^{p}-S_{a, c}^{r}\right)
$$

\section{Ex post survivability by cause of death}

To decompose ex post survivability by cause of death, we rewrite differences in remaining life expectancy, $R_{a}$ in equation (A.4), as the sum of differences in survival probabilities in succeeding ages:

$$
R_{a}^{p}-R_{a}^{r}=\sum_{j=a+1}^{\bar{a}}\left(\prod_{i=a+1}^{j}\left(1-M_{i}^{p}\right)-\prod_{i=a+1}^{j}\left(1-M_{i}^{r}\right)\right)=\sum_{j=a+1}^{\bar{a}}\left(\tilde{S}_{j}^{p}-\tilde{S}_{j}^{r}\right)
$$

where $\tilde{S}_{j} \equiv \prod_{i=a+1}^{j}\left(1-M_{i}\right)$ is the probability of surviving from age $a+1$ to past age $j$. As in equation (A.10), we can rewrite differences in survival as the sum of differences in cause-specific

survival probabilities $\tilde{S}_{j}^{p}-\tilde{S}_{j}^{r}=\sum_{c}\left(\tilde{S}_{j, c}^{p}-\tilde{S}_{j, c}^{r}\right)$, where the cause-specific survival probabilities reflect the probability that a person does not die from that particular disease during this age span. 
Inserting this into the expression for ex post survivability yields:

$$
\begin{aligned}
\sum_{a=\underline{a}}^{\bar{a}} \overline{\Delta M_{a}} \cdot \overline{S_{a}} \cdot\left(R_{a}^{p}-R_{a}^{r}\right) & =\sum_{a=\underline{a}}^{\bar{a}} \overline{\Delta M_{a}} \cdot \overline{S_{a}} \cdot \sum_{j=a+1}^{\bar{a}} \sum_{c}\left(\tilde{S}_{j, c}^{p}-\tilde{S}_{j, c}^{r}\right) \\
& =\sum_{a=\underline{a}}^{\bar{a}} \overline{\Delta M_{a}} \cdot \overline{S_{a}} \cdot \sum_{c}\left(\tilde{Q}_{j, c}^{r}-\tilde{Q}_{j, c}^{p}\right)
\end{aligned}
$$

where we have used $\tilde{Q}_{a, c}^{k}=\sum_{j=a+1}^{\bar{a}}\left(1-\tilde{S}_{j, c}^{k}\right)$ for $k=r, p$, which denotes expected lost life measured in years from age $a$ due to the risks of dying of cause $c$.

\section{Alternative definition of behavioral diseases}

We study a broader group of behavioral diseases than Case and Deaton (2015). We do this for two reasons: (1) to account for discrepancies in the coding of different diseases across countries, for example that deaths coded as poisonings in the US (ICD-10 codes X) are often coded as substance abuse disorders (ICD-10 codes F1) in Denmark and (2) to include chronic obstructive pulmonary disease (COPD), which is mainly caused by smoking. Figure A.3 presents cause-specific decompositions of changes in life expectancy inequality by income for Denmark, where we reclassify the diseases studied in Figure 2 of Case and Deaton (2015) as behavioral (poisoning, lung cancer, suicide, chronic liver disease, diabetes). The results are very similar to the original results, but other diseases now explain a larger fraction of the difference in survivability while behavioral diseases explain a smaller fraction. This difference is mainly driven by COPD, which we include in our definition of behavioral diseases and which is much more common among the poor than the rich.

\section{Relative changes in mortality rates and life expectancy}

Our main analysis focuses on absolute changes in mortality rates as is commonly done in the literature (Currie and Schwandt, 2016; Case and Deaton, 2015; Kinge et al., 2019). One may ask whether differential relative changes in mortality rates between rich and poor map one-to-

one into differential changes in their life expectancy. Below we show that this is not the case. 
Differences in survivability are still important and life expectancy may rise more for the rich than the poor even when relative changes in mortality rates are the same for both groups. We first augment our decomposition formula to study differences in relative changes in mortality rates. Using $\Delta M_{a} \equiv \frac{\Delta M_{a}}{M_{a}} \cdot M_{a}$, we may rewrite equation (3) in the main text as

$$
\Delta L E_{\underline{a}}^{r}-\Delta L E_{\underline{a}}^{p}=\sum_{a=\underline{a}}^{\bar{a}} \overline{X_{a}} \cdot\left(\frac{\Delta M_{a}^{p}}{M_{a}^{p}} \cdot M_{a}^{p}-\frac{\Delta M_{a}^{r}}{M_{a}^{r}} \cdot M_{a}^{r}\right)+\sum_{a=\underline{a}}^{\bar{a}} \overline{\Delta M_{a}} \cdot\left(X_{a}^{p}-X_{a}^{r}\right)
$$

By means of the same method as described in equations (A.7) and (A.8) above, this can be further decomposed into changes in relative mortality rates and differences in initial mortality rates:

$$
\begin{aligned}
\Delta L E_{\underline{a}}^{r}-\Delta L E_{\underline{a}}^{p}= & \sum_{a=\underline{a}}^{\bar{a}} \overline{X_{a}} \cdot \overline{M_{a}} \cdot\left(\frac{\Delta M_{a}^{p}}{M_{a}^{p}}-\frac{\Delta M_{a}^{r}}{M_{a}^{r}}\right) \\
& +\sum_{a=\underline{a}}^{\bar{a}} \overline{X_{a}} \cdot \overline{\left(\frac{\Delta M_{a}}{M_{a}}\right)} \cdot\left(M_{a}^{p}-M_{a}^{r}\right)+\sum_{a=\underline{a}}^{\bar{a}} \overline{\Delta M_{a}} \cdot\left(X_{a}^{p}-X_{a}^{r}\right)
\end{aligned}
$$

The first term in the decomposition can be interpreted as the change in life expectancy inequality that would occur if relative changes in mortality rates differed across income groups, but initial mortality rates were the same. The second term reflects that, if the number of individuals surviving to a given age is the same across income groups, the same percentage drop in mortality will increase the number of survivors at that age more for the poor than the rich, because the poor have higher baseline mortality. Hence, if baseline mortality is twice as large for the poor, the same percentage drop in mortality will lead to twice as many new survivors at that age. The third term, which is just the standard survivability term, reflects that fewer among the poor will survive to benefit from a reduction in mortality at a given age (ex ante survivability) and that for each new survivor at that age, the remaining life expectancy is lower for the poor than the rich (ex post survivability).

Importantly, this decomposition shows that regardless of whether we study absolute or relative mortality rate changes, we cannot tell whether inequality in life expectancy is increasing or decreasing on the basis of mortality rate changes alone. Even equal relative changes in mortality 
rates across income groups can lead to increasing life expectancy inequality because of differences in survivability. Table A.1 provides an empirical example. For males in Denmark, column (1) shows that mortality at age 85 has dropped by approximately $18 \%$ for both the rich and the poor. With initial mortality rates of 0.17 for the poor and 0.14 for the rich, see column (2), this means that among those surviving to age 85 , the share of individuals surviving beyond age 85 has increased more for the poor (3 percentage points) than the rich (2.5 percentage points). In isolation, this reduces inequality. However, as shown in column (3) of Table A.1, only $12 \%$ of the poor survive long enough to benefit from the reduction in mortality at age 85 versus $28 \%$ of the rich. In addition, for those who now survive beyond age 85, column (4) shows that the remaining life expectancy is 4.3 years for the poor compared to 4.8 years for the rich. These differences in survivability, reported in column (5), imply that the same relative change in mortality rates across income groups leads to twice the increase in life expectancy for the rich compared to the poor, as shown in column (6). Column (7) shows that the percentage increase in life expectancy is also highest for the rich.

\section{Accounting for income mobility}

We do not account for income mobility when computing life expectancy by income and this can lead to an upward bias in the estimated inequality and its trend over time (Kreiner et al., 2018). In Figure A.4, we account for income mobility in our main decomposition results by implementing a method from Kreiner et al. (2018) that simultaneously predicts income mobility and age-specific mortality rates. This method requires micro data on income and mortality, which we have for Denmark but not for the US. Therefore, Figure A.4 only plots results for Denmark.

As is well known, accounting for income mobility attenuates the rise in life expectancy inequality over time (Kreiner et al., 2018). For example, inequality in life expectancy for Danish males increases by 0.4 years during 2001-2014 when accounting for mobility, compared to 0.7 years when not accounting for mobility. However, the relative importance of differences in survivability in ex- 
plaining the rise in inequality does not change when we account for income mobility. Differences in survivability still explain all of the increase in inequality in Denmark.

\section{$9 \quad$ Disability-adjusted life expectancy}

Life expectancy is a summary measure of health in a population based on mortality. This does not account for the quality of life or burden of disease. For this reason, researchers and health organizations often compute health expectancies or disability-free life expectancies, which put lower weight on life years lived with disability. Figure A.5 displays our main decomposition results using disability-free life expectancy $D F L E$. We do this by applying a weight $\left(W_{a}\right)$, which measures the value of a year of life at each age. This is equivalent to the often-used Sullivan method (Sullivan, 1971).

$$
\begin{aligned}
D F L E_{\underline{a}} & =\underline{a}+W_{\underline{a}} \cdot\left(1-M_{\underline{a}}\right)+W_{\underline{a}+1} \cdot\left(1-M_{\underline{a}}\right) \cdot\left(1-M_{\underline{a}+1}\right)+\ldots \\
& =\underline{a}+\sum_{j=\underline{a}}^{\bar{a}} W_{j} \prod_{i=\underline{a}}^{j}\left(1-M_{i}\right)
\end{aligned}
$$

Differentiating with respect to mortality at a given age $a$ yields:

$$
\begin{aligned}
\Delta D F L E_{\underline{a}} & =-\Delta M_{a} \cdot \prod_{i=\underline{a}}^{a-1}\left(1-M_{i}\right) \cdot \sum_{j=a}^{\bar{a}} W_{j} \prod_{i=a+1}^{j}\left(1-M_{i}\right) \\
& =-\Delta M_{a} \cdot \prod_{i=\underline{a}}^{a-1}\left(1-M_{i}\right) \cdot\left(W_{a}+\sum_{j=a+1}^{\bar{a}} W_{j} \prod_{i=a+1}^{j}\left(1-M_{i}\right)\right) \\
& =-\Delta M_{a} \cdot \hat{X}_{a}
\end{aligned}
$$

where $\hat{X}_{a} \equiv S_{a} \cdot \hat{R}_{a}$ is disability-free survivability and $\hat{R}_{a} \equiv W_{a}+\sum_{j=a+1}^{\bar{a}} W_{j} \prod_{i=a+1}^{j}\left(1-M_{i}\right)$ is disability-free remaining life expectancy at age $a$. From (A.15), we follow the same steps as in (A.6)(A.8) above and decompose changes in inequality in disability-free life expectancy into contributions 
from differences in mortality rate changes and survivability:

$$
\Delta D F L E_{\underline{a}}^{r}-\Delta D F L E_{\underline{a}}^{p}=\sum_{a=\underline{a}}^{\bar{a}}\left[\left(\Delta M_{a}^{p}-\Delta M_{a}^{r}\right) \cdot \overline{\hat{X}_{a}}+\overline{\Delta M_{a}} \cdot\left(\hat{X}_{a}^{p}-\hat{X}_{a}^{r}\right)\right]
$$

To implement this decomposition, we need estimates of the disease weights $W_{a}$. We base these on WHO estimates of years lived with disability:

$$
W_{a}=1-\frac{Y L D_{a}}{P_{a}-D_{a}}
$$

where $Y L D_{a}$ is years lived with disability (summing over the entire population), $P_{a}$ is the population and $D_{a}$ is the number of deaths at age a. We obtain these three components by 5year age groups from WHO's Global Burden of Disease database, http://ghdx.healthdata.org/ gbd-results-tool. The weights measure the share of years lived at age a, $P_{a}-D_{a}$, that are spent without disability. WHO's years lived with disability estimates are based on the prevalence of certain diseases and the associated loss in life satisfaction, see https://www . who.int/healthinfo/ global_burden_disease/metrics_daly/en/. Note that the weights we calculate are population wide since we do not have information on burden of disease by income.

Figure A.5A plots the weights by age for Danish and US males and females in 2001. In both countries and for both sexes, the weights are around 0.85 at age 40 , suggesting that $85 \%$ of life years at age 40 are spent in full health. The weights decrease with age as the disease burden increases and at age 95 the weights are around 0.4. Hence, because of disability, a year of life at age 95 is worth less than half a year in full health.

Figure A.5B plots the decomposition results using the weights in Figure A.5A and the decomposition formula (A.16). Overall, the rise in life expectancy inequality is smaller when we adjust for disability. The smaller absolute numbers reflect that the disability adjustment downweights life years and thus reduces the absolute size of life expectancy, life expectancy inequality, and its trend 
over time. If we instead consider the relative importance of mortality rate changes and survivability, it is clear that adjusting for disability does not change the main results. Differences in survivability across the rich and poor still explain all of the increase in inequality in Denmark and between $25-50 \%$ of the increase in inequality in the US.

\section{Inequality trends in other Western European countries}

Our main results show that mortality trends favor the poor in Denmark, but the rich in the US. In Figure A.6, we apply our decomposition to education inequalities in life expectancy across a number of European countries. We estimate these using published mortality rates by education groups from Mackenbach et al. (2018). The education groups are based on the same International Standard Classification of Education 1997 (ISCED) codes we use for Denmark in Section 11 of this appendix. The data contains information on one-year mortality rates by 5-year age groups from age 40 to 75 and for various years between 1997 and 2013. The years available in the data differ across countries and therefore we report yearly changes in life expectancy. As the data is only available up until age 75 and only for 5-year age groups, we predict mortality rates using Gompertz approximations. After age 90, we apply population-wide mortality rates to all education groups, obtained from life tables estimated by the WHO (http://ghdx.healthdata.org/gbd-results-tool). Because the Gompertz approximations are based on 5-year age groups and only 7 observations per country, these results should be interpreted with caution. Also, when estimating life expectancies by education in Section 11 above, we account for changes in the composition of education over time. We cannot do this in the present analysis because we do not have information on the education shares over time in these countries.

Despite these caveats, the decomposition results across the nine Western European countries in Figure A.6 largely mimic the results for Denmark. Among males, all countries but one (Spain) have seen larger mortality rate reductions among the low-educated than among the high-educated. In six 
of the remaining eight countries, the favorable mortality trends for the low-educated are associated with increasing inequality in life expectancy due to differences in survivability. The results for females are more mixed. In seven out of nine countries, mortality trends have favored the loweducated, but in most of these cases this has led to reductions in life expectancy inequality. In two Nordic countries, Finland and Sweden, mortality rate changes have favored the high-educated and so have changes in life expectancy. 


\section{References}

Arriaga, E. E. (1984). Measuring and explaining the change in life expectancies. Demography 21(1), $83-96$.

Case, A. and A. Deaton (2015). Rising morbidity and mortality in midlife among white non-Hispanic Americans in the 21st century. Proceedings of the National Academy of Sciences 112(49), 1507815083.

Case, A. and A. Deaton (2017). Mortality and morbidity in the 21st century. BPEA Conference Drafts, 1-60.

Chetty, R., M. Stepner, S. Abraham, S. Lin, B. Scuderi, N. Turner, A. Bergeron, and D. Cutler (2016). The association between income and life expectancy in the United States, 2001-2014. JAMA - Journal of the American Medical Association 315(16), 1750-1766.

Currie, J. and H. Schwandt (2016). Inequality in mortality decreased among the young while increasing for older adults, 1990-2010. Science 352(6286), 708-712.

Kinge, J. M., J. H. Modalsli, S. Øverland, H. K. Gjessing, M. C. Tollånes, A. K. Knudsen, V. Skirbekk, B. H. Strand, S. E. Håberg, and S. E. Vollset (2019). Association of Household Income with Life Expectancy and Cause-Specific Mortality in Norway, 2005-2015. JAMA - Journal of the American Medical Association 321(19), 1916-1925.

Kreiner, C. T., T. H. Nielsen, and B. L. Serena (2018). Role of income mobility for the measurement of inequality in life expectancy. Proceedings of the National Academy of Sciences of the United States of America 115(46), 11754-11759.

Mackenbach, J. P., J. R. Valverde, B. Artnik, M. Bopp, H. Brønnum-Hansen, P. Deboosere, R. Kalediene, K. Kovács, M. Leinsalu, P. Martikainen, G. Menvielle, E. Regidor, J. Rychtaříková, M. Rodriguez-Sanz, P. Vineis, C. White, B. Wojtyniak, Y. Hu, and W. J. Nusselder (2018). Trends in health inequalities in 27 European countries. Proceedings of the National Academy of Sciences 115(25), 201800028.

Sullivan, D. F. (1971). A single index of mortality and morbidity. HSMHA health reports 86(4), $347-354$. 


\section{Appendix figures and tables}

Figure A.1: Life expectancy levels and decomposition of change in life expectancy inequality with Chetty et al. (2016) sample restrictions on Danish data
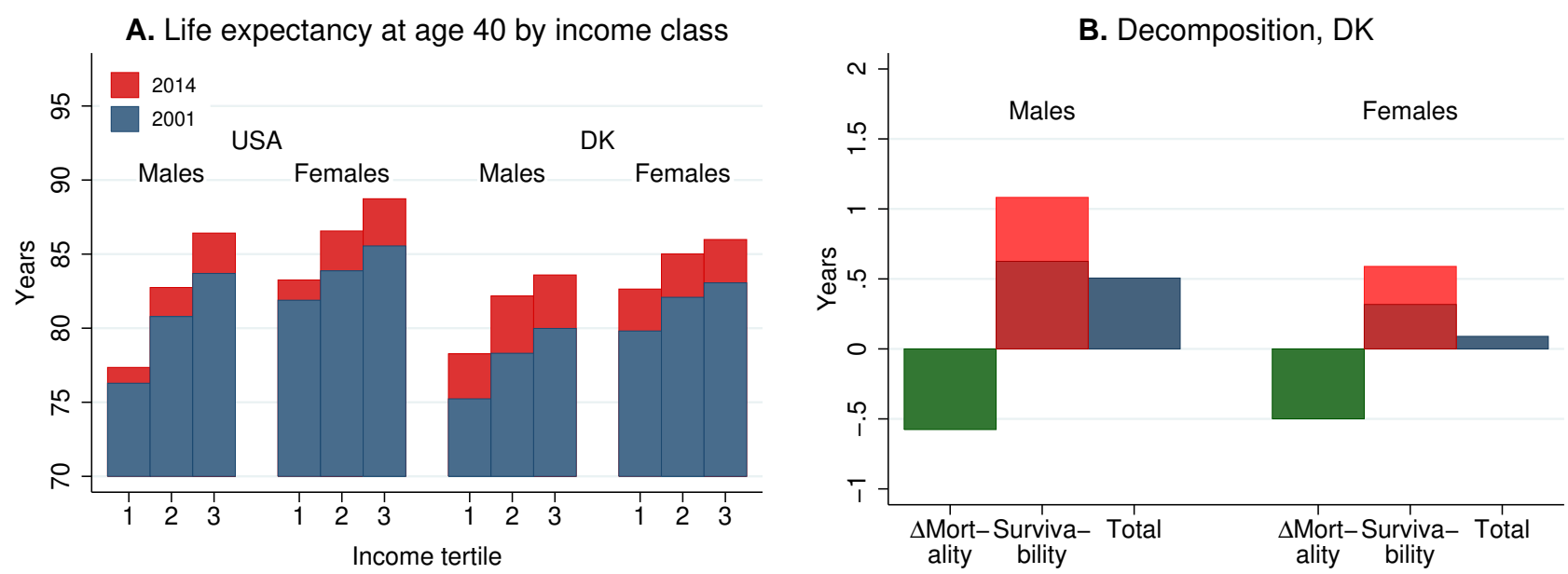

Notes: The two figures correspond to Figure 1 and Figure 3 in the paper with the only difference being that we have excluded individuals with zero or negative income and disability insurance recipients from the Danish data in order to to make the results more comparable across the two countries.

Table A.1: Effect of change in mortality at age 85 on life expectancy at age 40

\begin{tabular}{|c|c|c|c|c|c|c|c|}
\hline Rel. & $\begin{array}{c}\Delta \text { mort } \\
\frac{\Delta M_{85}}{M_{85}} \\
(1)\end{array}$ & $\begin{array}{l}\text { Mortality } \\
M_{85} \\
(2)\end{array}$ & $\begin{array}{c}\text { Survival } \\
\qquad S_{85} \\
(3)\end{array}$ & $\begin{array}{c}\text { Re. LE } \\
R_{85} \\
(4)\end{array}$ & $\begin{array}{l}\text { Survivability } \\
\qquad X_{85} \\
(5)=(3) \times(4)\end{array}$ & $\begin{array}{c}\Delta \text { Life Exp } \\
\Delta L E_{40} \\
(6)=(1) \times(2) \times(5)\end{array}$ & $\begin{array}{c}\text { Rel. } \Delta \text { Life Exp } \\
\frac{\Delta L E_{40}}{L E_{40}} \\
(7)\end{array}$ \\
\hline \multicolumn{8}{|c|}{ Denmark } \\
\hline Poor & -0.18 & 0.17 & 0.12 & 4.3 & 0.5 & 0.015 & 0.00021 \\
\hline Rich & -0.18 & 0.14 & 0.28 & 4.8 & 1.3 & 0.033 & 0.00041 \\
\hline
\end{tabular}

Notes: The table shows the effect of the observed same relative change in mortality rates of rich and poor 85 year old males on life expectancy at age 40. The table reports: (1) relative changes in mortality rates during 2001-2014, (2) initial mortality rates, (3) survival probabilities, $S_{85}$, up to age 85 , (4) remaining life expectancy after age 85 , $R_{85}$, (5) survivability at age $85, X_{85} \equiv S_{85} \cdot R_{85}$, (6) the effect on life expectancy at age 40 from the changes in mortality rates at age 85 , and (7) change in life expectancy relative to its level in 2001 . The change in life expectancy in column (6) is computed from the first-order approximation in equation (2). Survival probabilities and remaining life expectancies are calculated using the definitions reported below equation (2) and measured in 2001 in accordance with the first-order approximation. 
Figure A.2: Accuracy of first order approximation and decomposition of life expectancy inequality based on Arriaga age decomposition, rich - poor
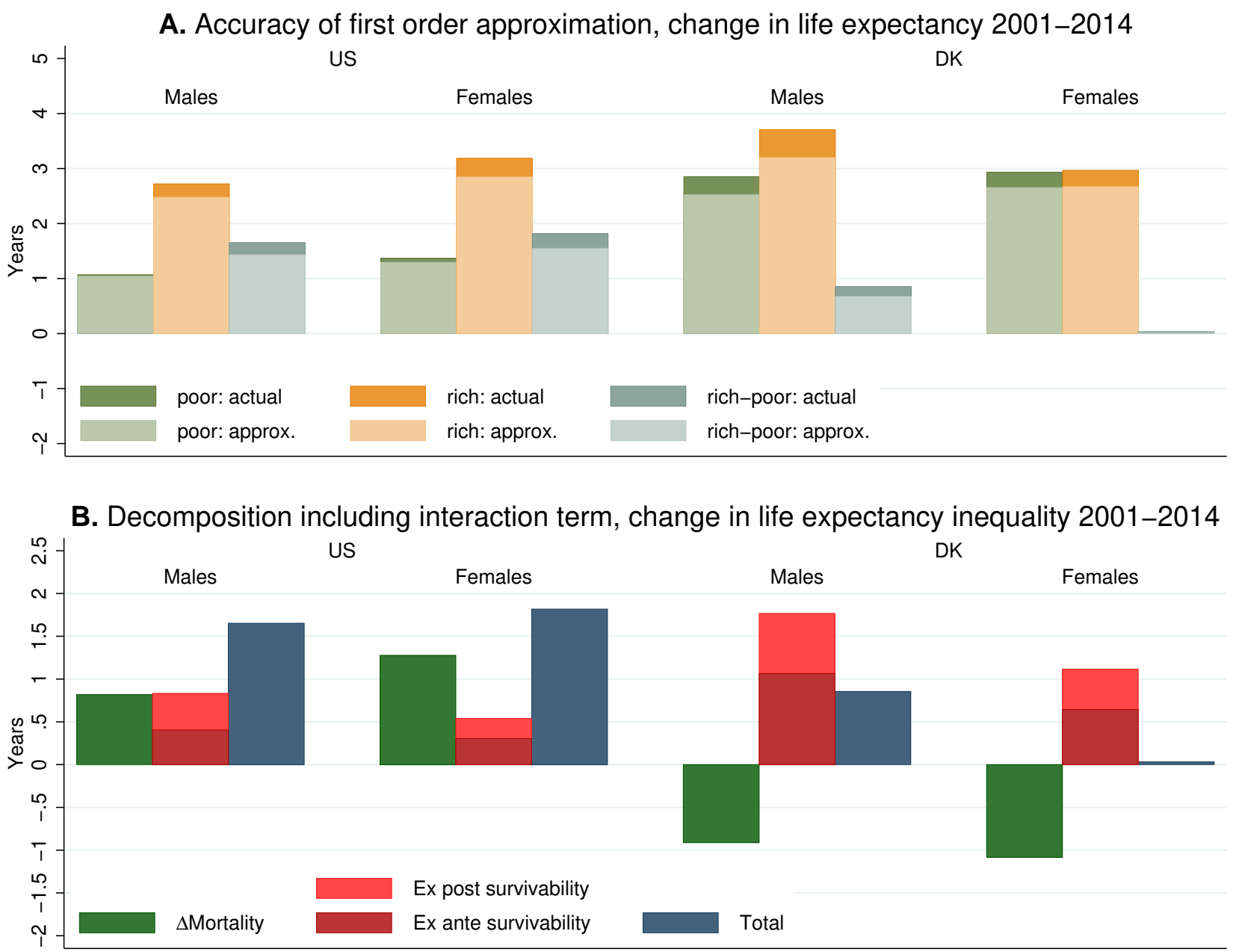

Notes: Panel A compares the changes in life expectancy at age 40 computed from the first order approximation in equation (2) to the actual changes in life expectancy. Panel B repeats the decomposition exercise in Figure 3, but based on an Arriaga age decomposition instead of the first-order approximation in equation (2). 
Figure A.3: Cause-specific decomposition of change in life expectancy inequality, rich - poor, using behavioral diseases from Case and Deaton (2015)

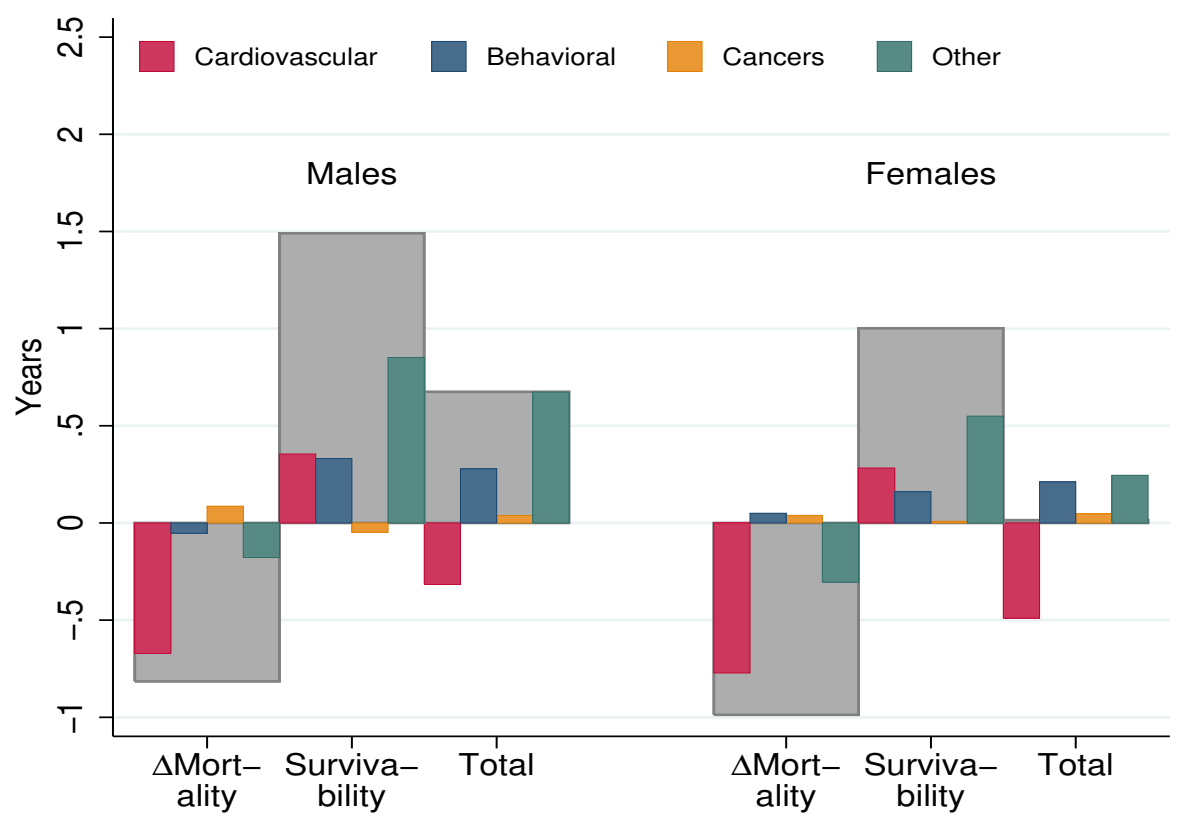

Notes: The figure is similar to Figure 4, but uses a slightly narrower definition of behavioral diseases (poisoning, lung cancer, suicide, chronic liver disease, diabetes) that corresponds to Figure 2 of Case and Deaton (2015). Life expectancy is measured at age 40 . 
Figure A.4: Decomposition of change in life expectancy inequality 2001-2014 when accounting for income mobility, rich-poor

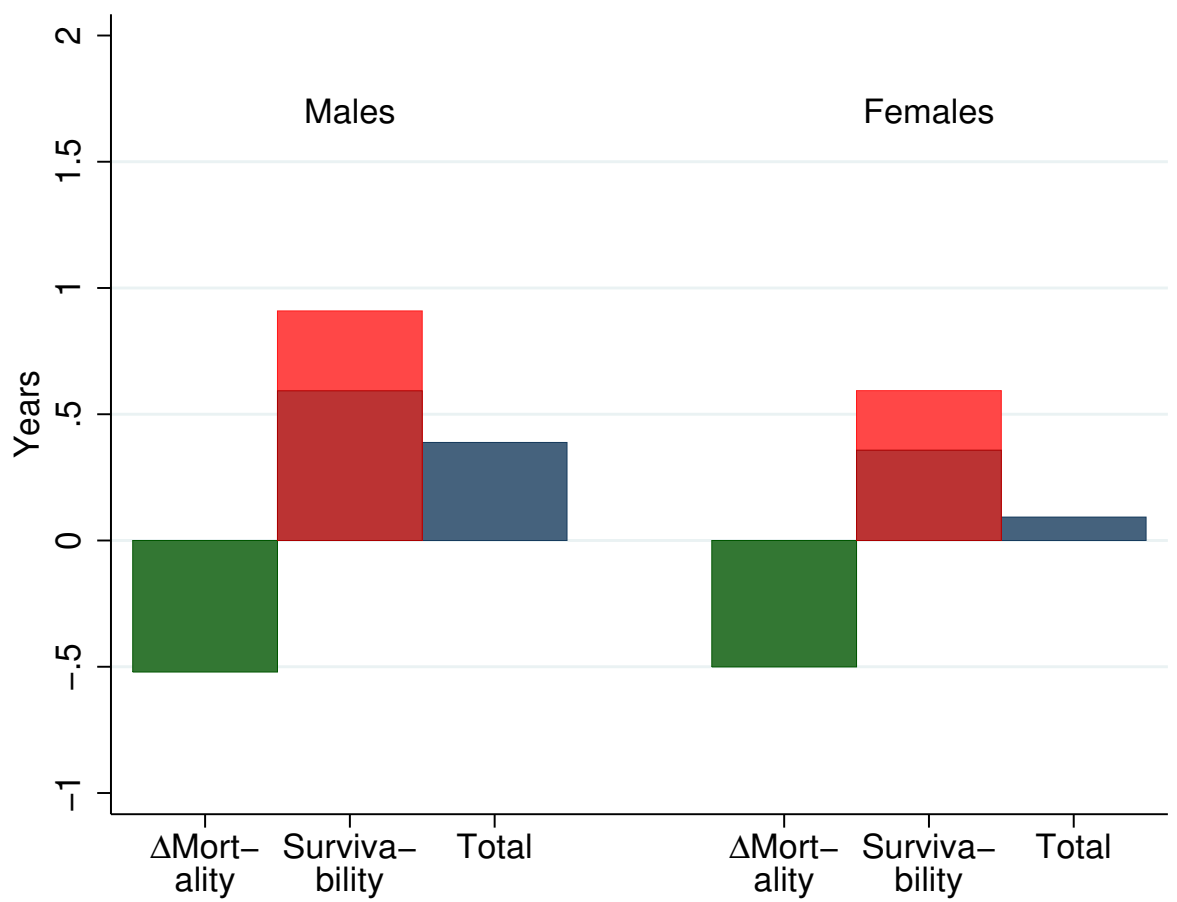

Notes: The figure corresponds to Figure 3, but here we account for income mobility in the decomposition results by implementing the method from Kreiner et al. (2018) that simultaneously predicts income mobility and age-specific mortality rates. Life expectancy is measured at age 40 . 
Figure A.5: Decomposition of change in disability-free life expectancy inequality over time
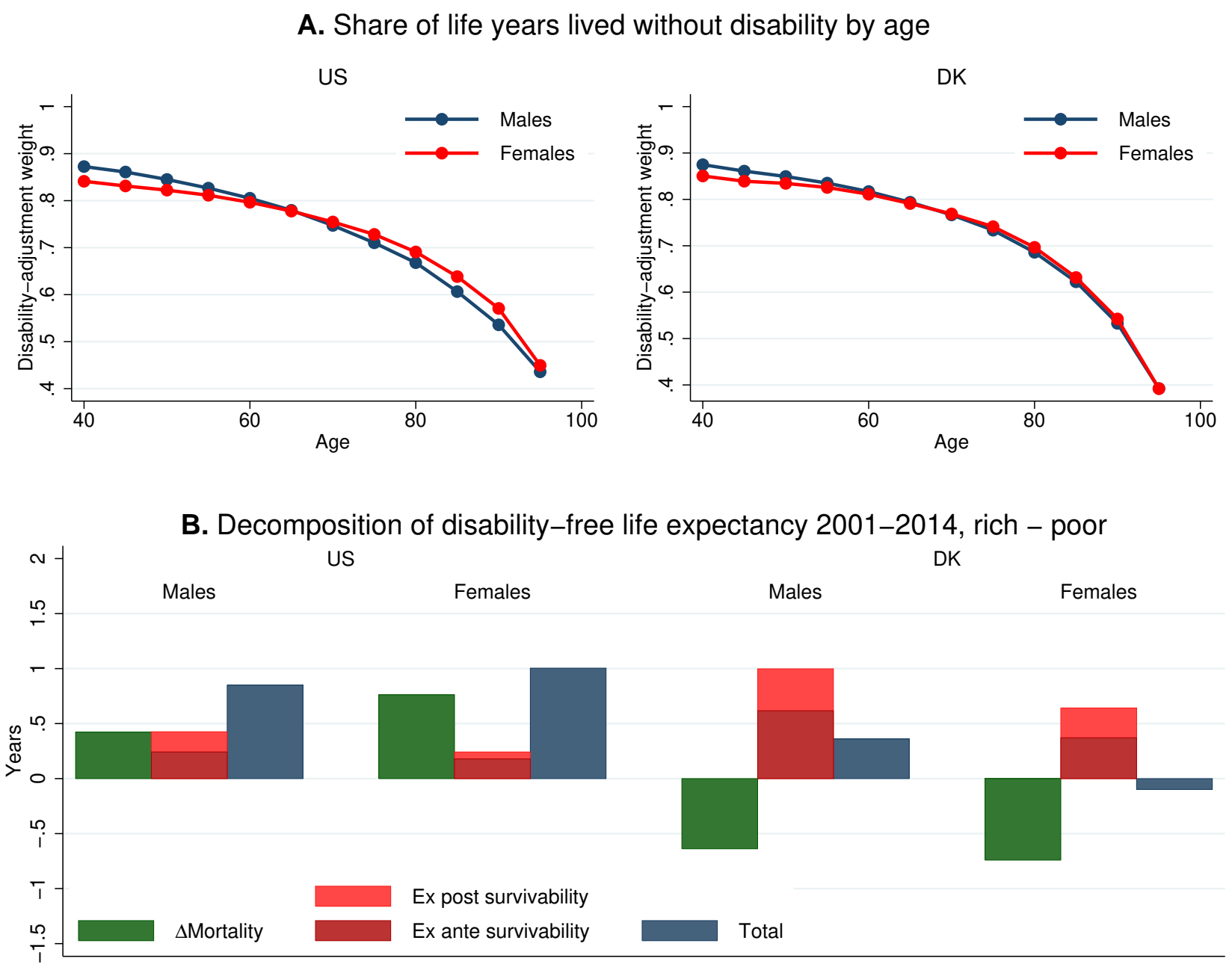

Notes: Panel A plots the weights applied in the disability-free life expectancy measure by age for Danish and US males and females in 2001. Each weight measures the average time spent in full health. Panel B plots the decomposition results using the weights in Panel A and the decomposition formula (A.16). Life expectancy is measured at age 40. 
Figure A.6: Decomposition of yearly change in life expectancy inequality by education for Western European countries, low-high education

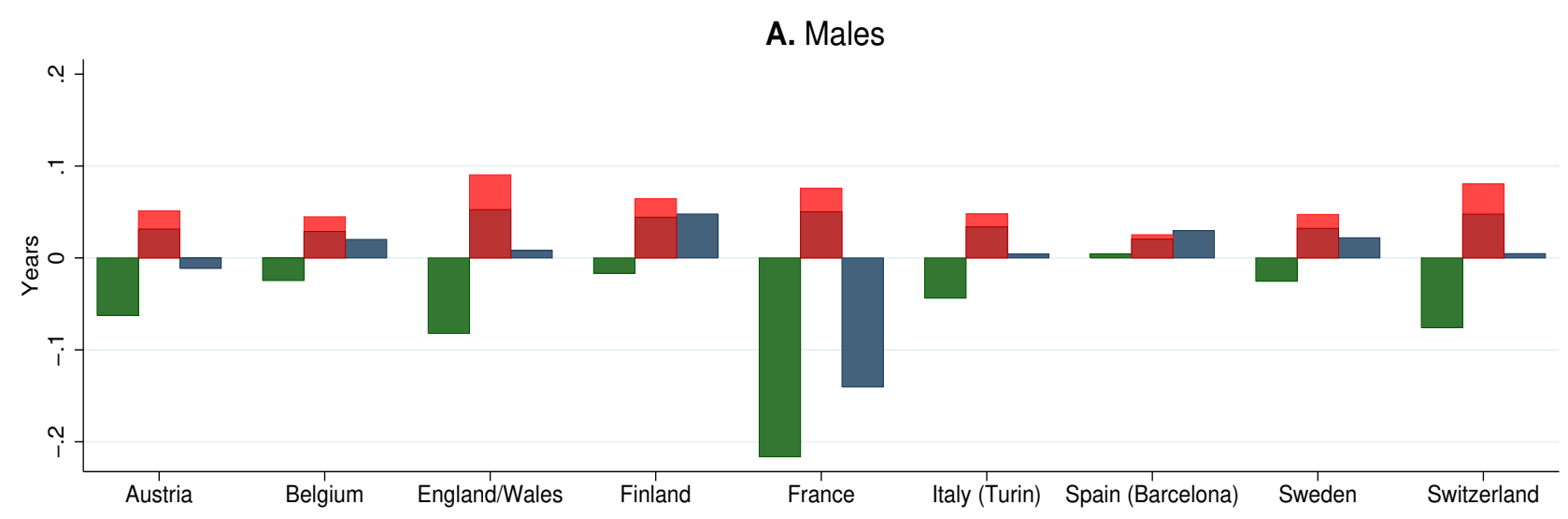

B. Females

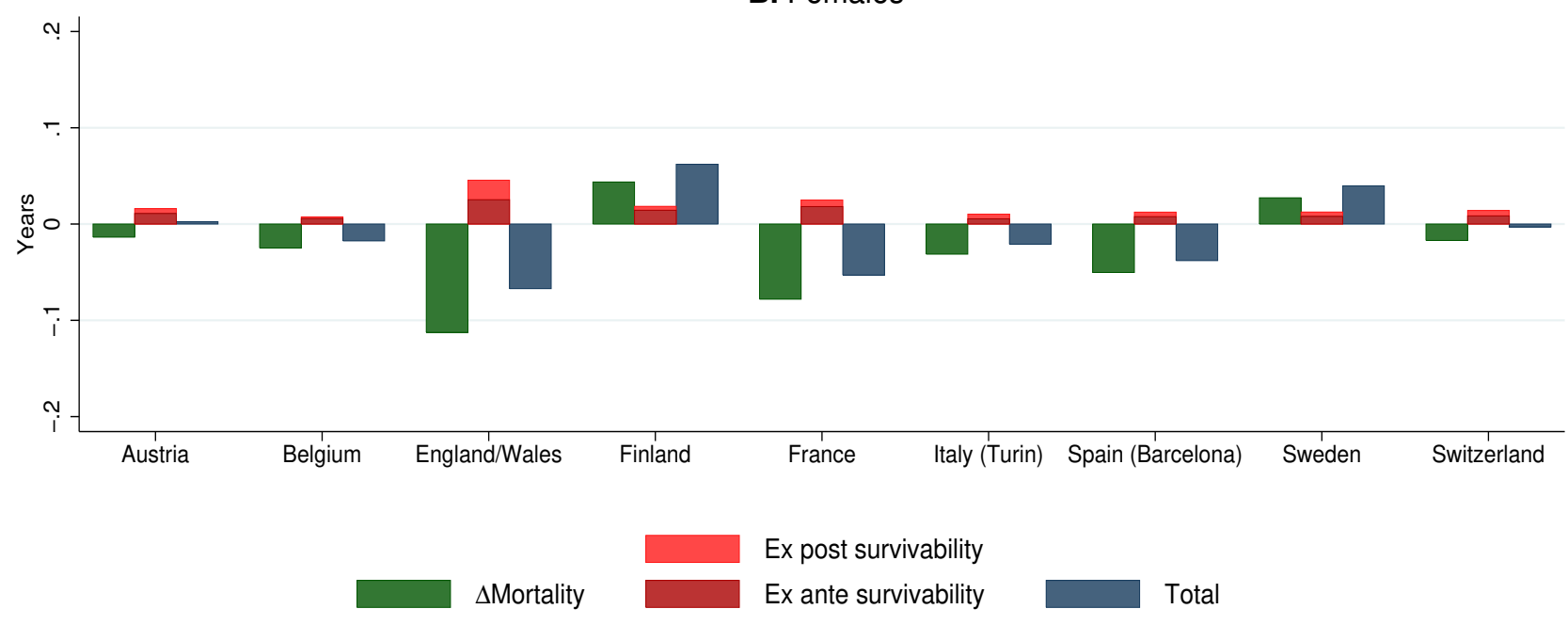

Notes: The figure shows the results from a decomposition of education inequalities in life expectancy across a number of European countries where information on educational attainment exists. The figure is comparable to Figure 3. Life expectancy is measured at age 40 . 
Figure A.7: Decomposition of change in life expectancy gap, females vs. males

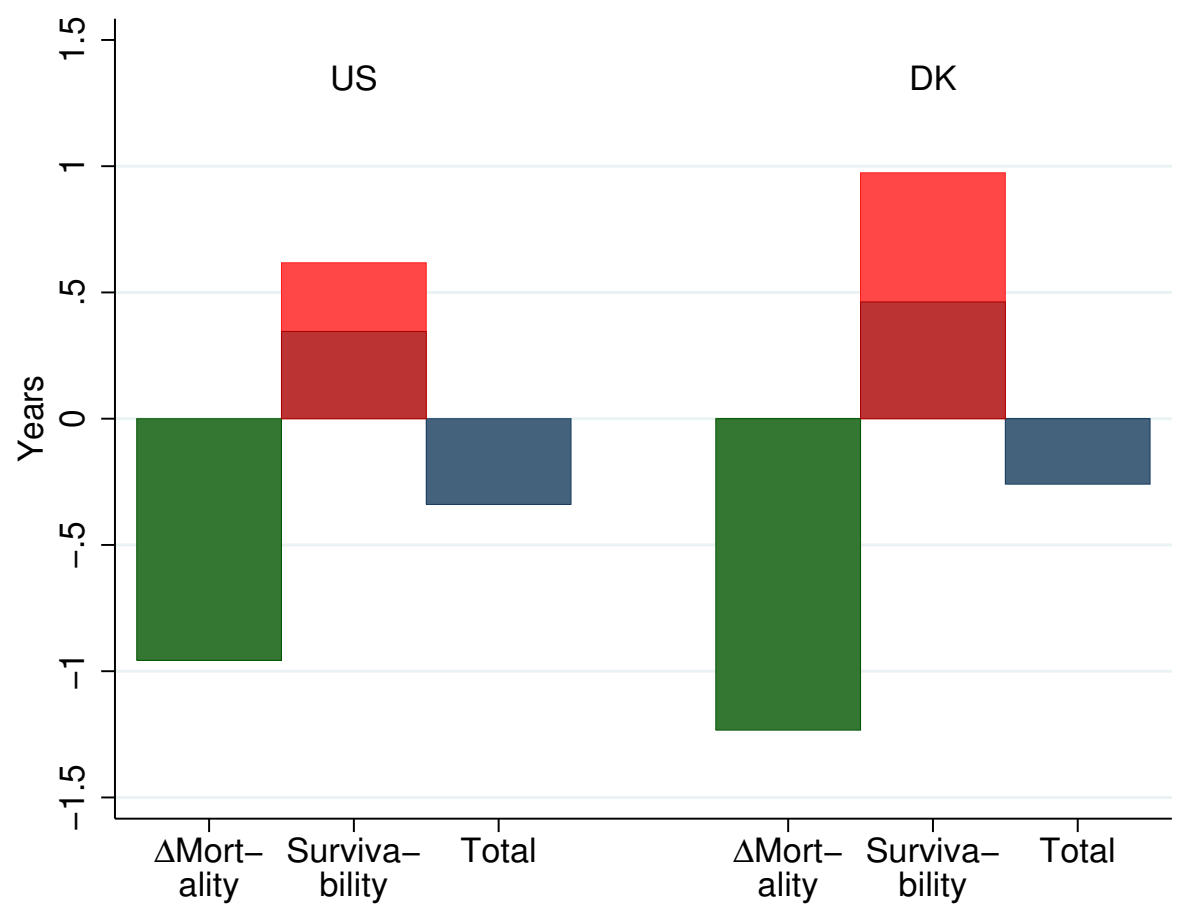

Notes: The figure shows the result from a decomposition of the change in the gender gap in life expectancy. The figure corresponds to the decomposition in Figure 3, but in this case showing the decomposition of the gender gap instead of the rich-poor gap. The decomposition is computed using equation (3). Life expectancy is measured at age 40. 
Figure A.8: Mortality declines of the poor needed to keep inequality constant
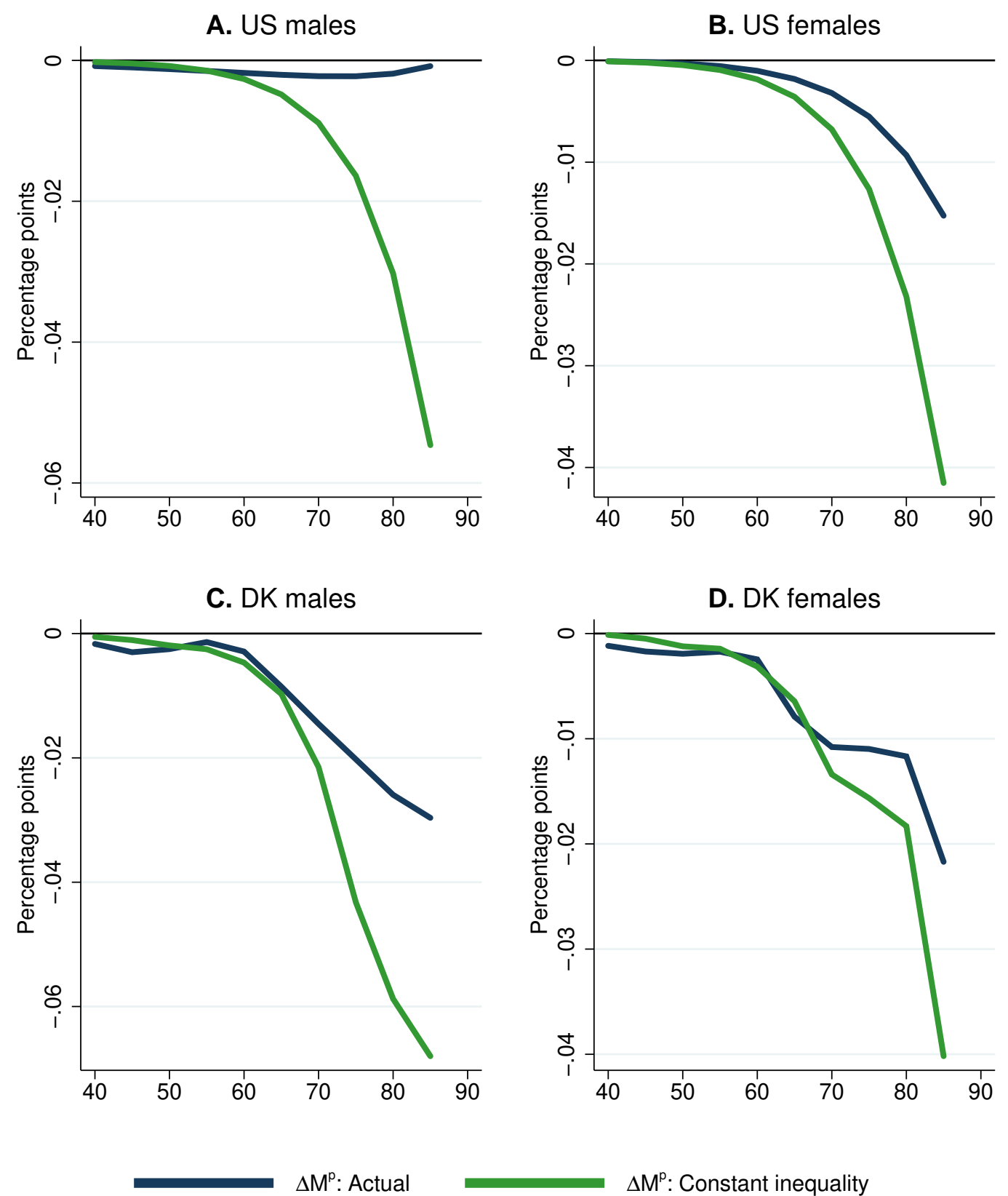

$\Delta \mathrm{M}^{\mathrm{p}}:$ Constant inequality

Notes: This figure illustrates how much mortality rates of the poor would need to decline, keeping the mortality changes of the rich at their actual levels, in order to keep life expectancy inequality unchanged (green line). The figure compares this to the actual declines in mortality among the poor (blue line). 
Figure A.9: Impact of a common one percentage point drop in different causes of death on life expectancy inequality

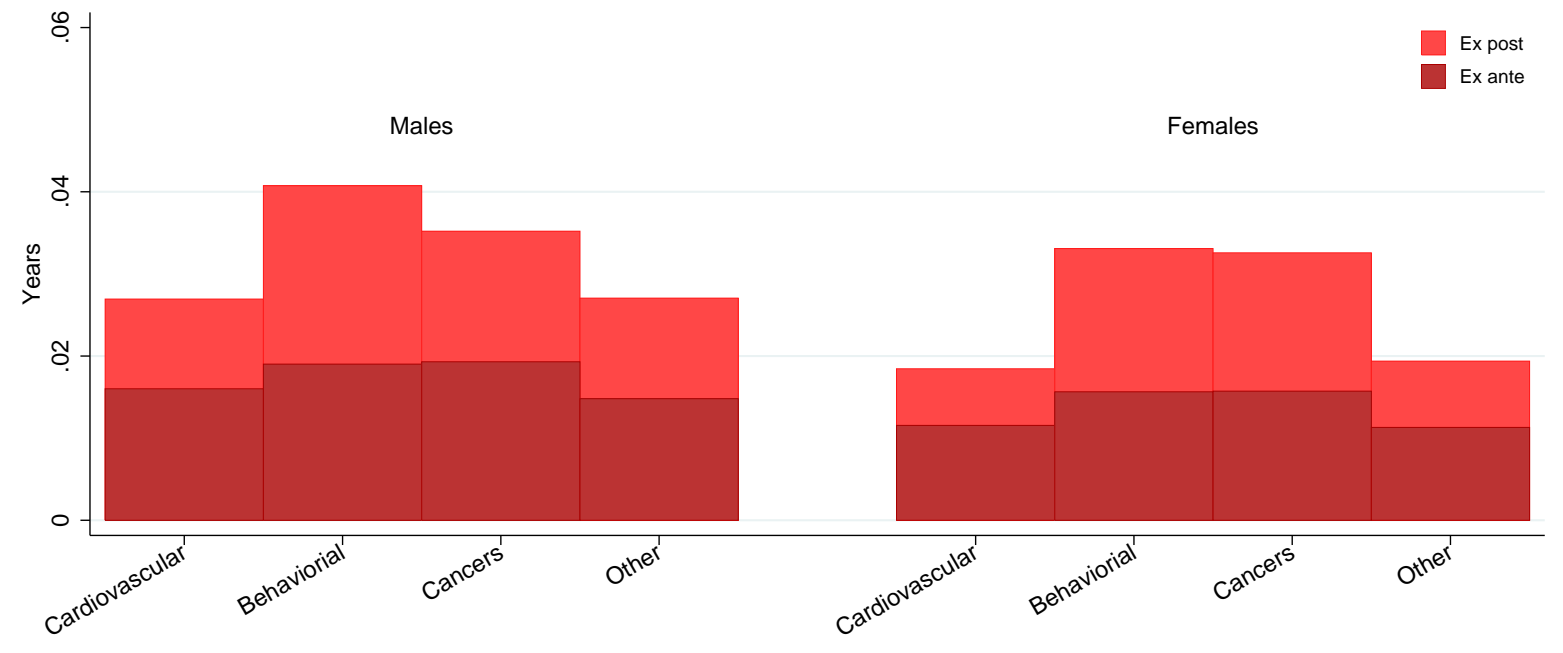

Notes: This figure shows the impact on the rich-poor gap in life expectancy at age 40 from a simulation of a common one-percentage point decline in overall mortality for different causes of death. The mortality reduction at a given age is distributed according to the age-distribution of mortality for the different death causes. We use equation (5) to compute the effects (note that the first term in this equation is zero when studying the same mortality rate drop for poor and rich). 